\title{
Physics-Based Predictions for Coherent Change Detection Using X-Band Synthetic Aperture Radar
}

\author{
Mark L. Williams \\ Imaging Radar Systems Group, Intelligence, Surveillance \& Reconnaissance Division, Defence Science \& Technology Organisation, \\ P.O. Box 1500, Edinburgh, SA 5111, Australia \\ Email:mark.williams@dsto.defence.gov.au \\ Mark Preiss \\ Imaging Radar Systems Group, Intelligence, Surveillance \& Reconnaissance Division, Defence Science \& Technology Organisation, \\ P.O. Box 1500, Edinburgh, SA 5111, Australia \\ Email: mark.preiss@dsto.defence.gov.au
}

Received 29 July 2004; Revised 9 December 2004

\begin{abstract}
A theoretical model is developed to describe the interferometric coherency between pairs of SAR images of rough soil surfaces. The model is derived using a dyadic form for surface reflectivity in the Kirchhoff approximation. This permits the combination of Kirchhoff theory and spotlight synthetic aperture radar (SAR) image formation theory. The resulting model is used to describe the interferometric coherency between pairs of SAR images of rough soil surfaces. The theoretical model is applied to SAR images formed before and after surface changes observed by a repeat-pass SAR system. The change in surface associated with a tyre track following vehicle passage is modelled and SAR coherency estimates are obtained. Predicted coherency distributions for both the change and no-change scenarios are used to estimate receiver operator curves for the detection of the changes using a highresolution, X-band SAR system.
\end{abstract}

Keywords and phrases: synthetic aperture radar, coherent change detection, surface scattering.

\section{INTRODUCTION}

Coherent change detection (CCD) using synthetic aperture radar (SAR) images from repeated apertures is an interferometric SAR technique and an increasingly important and rapidly developing area of research $[1,2,3]$. CCD techniques may be used to detect scene disturbances that occur between repeated SAR observations, which would otherwise remain undetected by incoherent change detection techniques. This is because CCD techniques are sensitive to the change in interferometric phase associated with scene changes, and not simply the change in backscatter intensity.

In order to predict the utility of future SAR systems designed to exploit interferometric coherence, it is necessary to understand the dependence of coherence upon both scene characteristics and imaging parameters. In practice, natural weathering processes, such as sun, wind, and rain, will induce change in a scene between observations, resulting in loss of coherence, and these processes must also be understood.

This is an open access article distributed under the Creative Commons Attribution License, which permits unrestricted use, distribution, and reproduction in any medium, provided the original work is properly cited.
Indeed, it has been observed that even at short time intervals coherence at X-band can be substantially destroyed by plant motion over densely vegetated areas [4]. On the other hand, under benign conditions, high coherence has been observed to persist for several days over rough surfaces. Shorter vegetation is less prone to wind-induced motion, and can be crushed by the passage of vehicles [3]. The detection of change under such conditions deserves careful consideration. Thus, at X-band, at least SAR CCD is likely to be most useful over sparsely vegetated or bare soil surfaces, in particular, where these surfaces are in clear view to the radar, which is most likely to mean a weathered, artificial surface, rather than a natural surface. Thus, change detection at X-band for bare, rough surfaces is also important and is considered here.

We report a theoretical approach designed to model the detection of changes in soil surfaces. The theory incorporates both a physical model for surface scattering (Kirchhoff theory, see, e.g., $[5,6,7])$ and a model for spotlight SAR signal processing $[8,9]$. We derive a dyadic form for surface reflectivity in the Kirchhoff approximation. Using this dyadic reflectivity, a direct, rigorous connection is drawn between spotlight SAR pixel values and surface reflectivity in the tangent plane approximation. 
The expression for SAR pixel values is then used to obtain another for coherency between SAR images. Coherency is expressed in a manner similar to that of Zebker and Villasenor [10], but extends that work by incorporating a deterministic, physical theory of surface scattering rather than a statistical model of surface reflectivity correlation. This extension permits the theoretical study of the dependence of coherency upon physical surface change. The theoretical model reveals that coherence between images formed from separate apertures may depend not only upon scene change, but also upon the spatial correlation in reflectivity of the imaged surface, in addition to the separation of the apertures in $k$-space.

The current trend in high-frequency (X-band) airborne and spaceborne SAR systems is towards ever-increasing resolution $[11,12]$. Submetre resolution is now commonplace and future developments will no doubt see improvements in resolution towards the diffraction limit. We demonstrate the utility of the theoretical model by performing numerical calculations designed to predict the performance of a very-highresolution SAR system capable of generating $0.15 \mathrm{~m}$ resolution spotlight SAR imagery. Modelled scene changes are appropriate for tyre tracks generated by the passage of a vehicle across a rough soil surface. The sensitivity of the very-highresolution SAR CCD instrument is determined by calculating the distribution of coherence in both change and nochange scenarios. Coherence distributions are used to estimate receiver operator curves (ROCs) for different levels of change.

In the following section, we describe the derivation of the dyadic surface reflectivity in the Kirchhoff approximation, and the combination of surface scattering theory with spotlight SAR imaging theory to obtain estimates of coherence for SAR images of rough surfaces. We then proceed to describe calculations designed to assess the performance of a very-high-resolution SAR CCD instrument. Results of these calculations are discussed in the next section, and finally we comment on both the utility of the model and on the use of future SAR CCD systems.

\section{SURFACE SCATTERING, SPOTLIGHT SAR, AND SAR CCD: THEORY}

\subsection{Surface reflectivity and the received signal}

We consider spotlight SAR images formed using repeated transmission of a radar pulse described by

$$
p(\hat{t})=\int P(\omega) \exp (-i \omega \hat{t}) d \omega .
$$

In (1), $\hat{t}=t-n T$ is the so-called "fast" time after pulse transmission of the $n^{\text {th }}$ pulse at time $n T[8,9]$ (the leading phase of the $n^{\text {th }}$ pulse has been omitted). The received signal at alongtrack "slow" time $u$,

$$
\begin{aligned}
& \underline{\underline{s}}(u, \hat{t}) \\
& \quad=\int_{-\infty}^{+\infty} P(\omega) \int_{\Gamma} \underline{\underline{F}}(\omega, \mathbf{s}, u) \exp (i \omega \tau(u, \mathbf{s})) d \Gamma \exp (-i \omega \hat{t}) d \omega
\end{aligned}
$$

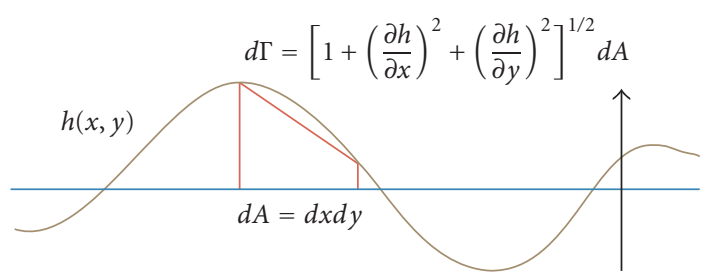

FIGURE 1: Integration over the imaging plane.

is used to form the image. Equation (2) defines the polarimetric, reflectivity density function $\underline{\underline{F}}(\omega, \mathbf{s}, u)$ measured during the SAR observation. In repeat-pass SAR CCD, a pair of images is formed and on each occasion an observation is made of the reflectivity function: changes to the scene result in changes to this function upon which the coherency between images will depend.

In (2), $\mathbf{s}=\{x, y, h(x, y)\}$ is a point on a real, rough surface of integration, and $d \Gamma$ is a differential element of $\Gamma$; the illuminated area of that surface (see Figure 1). The time delay

$$
\tau(u, \mathbf{s})=\frac{2|\mathbf{r}(u)-\mathbf{s}|}{c}
$$

( $c$ is the speed of light) is the signal travel time between antenna phase centre position $\mathbf{r}(u)$ and the surface point $\mathbf{s}$ in the so-called "stop-start" approximation $[8,9]$. For simplicity, the effects of antenna and range gains have been incorporated into the reflectivity density.

For a narrow bandwidth system, the surface reflectivity is assumed constant at the centre frequency, $\omega_{o}=c / k_{o}$, and the received signal in (2) is approximated by

$$
\underline{\underline{s}}(u, \hat{t})=\int_{\Gamma} \underline{\underline{F}}\left(\omega_{o}, \mathbf{s}, u\right) p(\hat{t}-\tau(u, \mathbf{s})) d \Gamma .
$$

In (4), each elemental area of surface reflects back the original pulse with delay $\tau(u, s)$ and polarimetric amplitude $\underline{F}\left(\omega_{o}, \mathbf{s}, u\right) d \Gamma$. Taking a flat plane of integration [13] (rather than the undulating, rough surface, Figure 1) and remaining in the narrow bandwidth approximation,

$$
\begin{gathered}
\underline{\underline{s}}(u, \hat{t})=\iint_{S} \underline{\underline{\Phi}}\left(\omega_{o}, x, y, u\right) p(\hat{t}-\tau(x, y, u)) d x d y, \\
\underline{\underline{\Phi}}\left(\omega_{o}, x, y, u\right)=\underline{\underline{F}}\left(\omega_{o}, \mathbf{s}, u\right)\left[1+\left(\frac{\partial h}{\partial x}\right)^{2}+\left(\frac{\partial h}{\partial y}\right)^{2}\right]^{1 / 2}
\end{gathered}
$$

Given a suitable expression for the reflectivity density $\underline{\underline{F}}(\omega, \mathbf{s}, u),(5)$ is in a form suitable for considering in the polar format algorithm (PFA) $[8,9]$.

\subsection{Surface reflectivity in the Kirchhoff approximation}

Following the application of Green's dyadic theorem $[5,6,7]$, the harmonic scattered field at a single frequency $\omega_{o}$ may be written as a surface integral involving surface fields and the 
free-space dyadic Green's function $\underline{\underline{G}}$ :

$$
\begin{aligned}
\mathbf{E}_{s}(\mathbf{r})=\int_{\Gamma}[\hat{\mathbf{n}}(\mathbf{s}) \times \nabla \times \mathbf{E}(\mathbf{s})] \cdot \underline{\underline{G}}(\mathbf{s}, \mathbf{r}) \\
+[\hat{\mathbf{n}}(\mathbf{s}) \times \mathbf{E}(\mathbf{s})] \cdot \nabla \times \underline{\underline{G}}(\mathbf{s}, \mathbf{r}) d \Gamma,
\end{aligned}
$$

where $\mathbf{E}(\mathbf{s})$ is the field at the surface and $\hat{\mathbf{n}}(\mathbf{s})$ is the outward unit vector normal to the surface at $\mathbf{s}$. Writing for the pulse spectrum in (2), $P(\omega)=P \delta\left(\omega-\omega_{o}\right)$ yields the harmonic received signal

$$
\underline{\underline{s}}(u, \hat{t})=\exp \left(-i \omega_{o} \hat{t}\right) \int_{\Gamma} P \underline{\underline{F}}\left(\omega_{o}, \mathbf{s}, u\right) \exp \left(i \omega_{o} \tau(u, \mathbf{s})\right) d \Gamma
$$

and making explicit the range attenuation in the reverse direction in the (hitherto hidden) antenna gain function,

$$
\begin{aligned}
& \underline{s}(u, \hat{t}) \\
& \quad=\exp \left(-i \omega_{o} \hat{t}\right) \int_{\Gamma}\left[P \underline{\underline{F}}\left(\omega_{o}, \mathbf{s}, u\right) \frac{\exp \left(i 2 k_{o}|\mathbf{r}(u)-\mathbf{s}|\right)}{|\mathbf{r}(u)-\mathbf{s}|}\right] d \Gamma .
\end{aligned}
$$

Comparison of (6) with (8) reveals that

$$
\begin{aligned}
\frac{\exp \left(i 2 k_{o}|\mathbf{r}-\mathbf{s}|\right)}{|\mathbf{r}-\mathbf{s}|} \underline{\underline{F}}\left(\omega_{o}, \mathbf{s}, u\right) \cdot \mathbf{E}_{i} \\
=[\hat{\mathbf{n}}(\mathbf{s}) \times \nabla \times \mathbf{E}(\mathbf{s})] \cdot \underline{\underline{G}}(\mathbf{s}, \mathbf{r}) \\
\quad+[\hat{\mathbf{n}}(\mathbf{s}) \times \mathbf{E}(\mathbf{s})] \cdot \nabla \times \underline{\underline{G}}(\mathbf{s}, \mathbf{r}) .
\end{aligned}
$$

In (9), $\mathbf{E}_{i}$, the incident field strength at the surface, contains the range attenuation from transmitter to surface point, and the harmonic term has been omitted, as it has been in (6). To make use of (9), the tangent plane approximation is employed. We cast (6) in a more familiar form $[6,(4.12)]$ :

$$
\begin{aligned}
\mathbf{E}_{s}(\mathbf{r})= & i k_{o} \frac{\exp \left(i k_{o} r\right)}{4 \pi r}\left[\hat{\mathbf{h}}_{s} \hat{\mathbf{h}}_{s}+\hat{\mathbf{v}}_{s} \hat{\mathbf{v}}_{s}\right] \times \int_{\Gamma} \eta[\hat{\mathbf{n}} \times \mathbf{H}(\mathbf{s})] \\
& +\left[\hat{\mathbf{k}}_{s} \times \hat{\mathbf{n}} \times \mathbf{E}(\mathbf{s})\right] \exp \left(-i k_{o} \hat{\mathbf{k}}_{s} \cdot \mathbf{s}\right) d \Gamma
\end{aligned}
$$

Consideration of (6), (9), and (10) reveals the connection between the Kirchhoff surface reflectivity density and the surface tangential fields to be

$$
\begin{aligned}
& \underline{\underline{F}}\left(k_{o}, \mathbf{s}, \mathbf{r}\right) \cdot \mathbf{E}_{i} \\
& \quad=\frac{i k_{o}}{4 \pi}\left[\underline{\underline{I}}-\hat{\mathbf{k}}_{s} \hat{\mathbf{k}}_{s}\right] \cdot\left\{\eta[\hat{\mathbf{n}} \times \mathbf{H}(\mathbf{s})]+\left[\hat{\mathbf{k}}_{s} \times \hat{\mathbf{n}} \times \mathbf{E}(\mathbf{s})\right]\right\} .
\end{aligned}
$$

The surface tangential fields in the Kirchhoff approximation $[5,6,7]$ are (in this notation)

$$
\begin{aligned}
\hat{\mathbf{n}} \times \mathbf{E}(\mathbf{s})=\{ & \left(1+R_{h}\right)\left(\hat{\mathbf{n}} \times \widehat{\mathbf{h}}_{i l}\right) \hat{\mathbf{h}}_{i l} \\
& \left.-\left(1-R_{v}\right)\left(\hat{\mathbf{n}} \cdot \hat{\mathbf{k}}_{i}\right) \hat{\mathbf{h}}_{i} / \widehat{\mathbf{V}}_{i l}\right\} \cdot \mathbf{E}_{i} \exp \left(i \mathbf{k}_{i} \cdot \mathbf{s}\right),
\end{aligned}
$$

$$
\begin{aligned}
-\eta[\hat{\mathbf{n}} \times \mathbf{H}(\mathbf{s})]= & \left\{\left(1+R_{v}\right)\left(\hat{\mathbf{n}} \times \hat{\mathbf{h}}_{i l}\right) \hat{\mathbf{v}}_{i l}\right. \\
& \left.+\left(1-R_{h}\right)\left(\hat{\mathbf{n}} \cdot \hat{\mathbf{k}}_{i}\right) \hat{\mathbf{h}}_{i} \hat{\mathbf{h}}_{i l}\right\} \cdot \mathbf{E}_{i} \exp \left(i \mathbf{k}_{i} \cdot \mathbf{s}\right) .
\end{aligned}
$$

In (12) and (13), the quantities $R_{h}$ and $R_{v}$ are the local Fresnel reflection coefficients which depend upon the local incidence angle, as do the local polarisation vectors $\hat{\mathbf{h}}_{i l}$ and $\hat{\mathbf{v}}_{i l}$. After a little vector algebra (see Appendix A), it may be shown that

$$
\begin{aligned}
\underline{\underline{F}}(k, \mathbf{s}, \mathbf{r}) \equiv & \underline{\underline{F}}\left(\omega, \mathbf{s}, u_{p}\right) \\
= & \frac{i \omega}{2 \pi c}\left(\hat{\mathbf{n}}(\mathbf{s}) \cdot \widehat{\mathbf{k}}_{i}\left(u_{p}\right)\right) \\
& \times\left[\begin{array}{c}
R_{h}\left(\omega, \mathbf{s}, u_{p}\right) \hat{\mathbf{h}}_{i l}\left(\mathbf{s}, u_{p}\right) \hat{\mathbf{h}}_{i l}\left(\mathbf{s}, u_{p}\right) \\
-R_{v}\left(\omega, \mathbf{s}, u_{p}\right) \widehat{\mathbf{v}}_{i l}\left(\mathbf{s}, u_{p}\right) \widehat{\mathbf{v}}_{i l}\left(\mathbf{s}, u_{p}\right)
\end{array}\right],
\end{aligned}
$$

which defines the dyadic surface backscatter reflectivity density in the tangent plane approximation using local Fresnel reflection coefficients and polarisation vectors. The derivation of (14) is reported in detail in Appendix A. Expressing the tangential surface fields in this way permits the use of (5) in the polar format algorithm in order to obtain an expression for spotlight SAR pixel values in the Kirchhoff approximation.

\subsection{Spotlight SAR pixels and coherence}

We consider formation of the $p^{\text {th }}$ spotlight SAR image using the polar format algorithm (PFA) and a transmitted linear FM chirp pulse. A complete discussion of the derivation of the relationship between spotlight SAR pixel values and surface properties may be found in Appendix B. The description of SAR image formation using the $\mathrm{P} f a$ given in $[8,9]$ is followed for a differential surface element. The signal is then integrated over the surface. For an unweighted rectangular aperture in the narrow bandwidth and aperture approximations (see Appendix B), we find the polarimetric SAR pixel at $\mathbf{r}^{\prime}=\left(x^{\prime}, y^{\prime}\right)$, for incidence angle $\theta_{p}$, to be

$$
\begin{aligned}
\underline{\underline{\Omega}}_{p}\left(\mathbf{r}^{\prime}\right)= & \iint_{S} \Phi_{p}\left(\omega_{p}, \mathbf{r}, u_{o p}\right) R_{p}\left(\mathbf{r}, \mathbf{r}^{\prime}\right) \\
& \times \exp \left(i \phi_{p}\left(\mathbf{r}, \mathbf{r}^{\prime}\right)\right) d x d y, \\
\phi_{p}\left(\mathbf{r}, \mathbf{r}^{\prime}\right)= & 2 k_{p} \sin \theta_{p}\left(\mathbf{r}^{\prime}-\mathbf{r}-\frac{\hat{\mathbf{y}}_{p} h_{p}(\mathbf{r})}{\tan \theta_{p}}\right) \cdot \hat{\mathbf{y}}_{p}, \\
R_{p}= & \operatorname{Sinc}\left[\Delta \kappa_{x p}\left(x^{\prime}-x\right)\right] \\
& \times \operatorname{Sinc}\left[\Delta \kappa_{y p}\left(y^{\prime}-y-\frac{h(x, y)}{\tan \theta_{p}}\right)\right],
\end{aligned}
$$


where $u_{o p}$ is the centre of the $p^{\text {th }}$ aperture and where $\hat{\mathbf{y}}_{p}$ is a unit vector in the ground range direction. Equation (15) makes explicit the connection with [10] except that here a rigorous connection is made between the scattering theory and the surface reflectivity. In [10], the authors treat $\underline{\underline{\Phi}}_{p}$ as a stochastic variable on a flat surface with an infinitely narrow correlation function; this is not the case in the tangent plane approximation for random rough surfaces with finite correlation lengths.

Formal expressions are simplified in the narrow bandwidth approximation (NBA) and its use, where appropriate, is therefore desirable. Validity of the Kirchhoff approximation is assumed. For good approximation, backscatter reflectivity is linear in frequency and expansion of the reflectivity about the centre frequency reveals the NBA to be a limit upon the fractional bandwidth of the SAR system. For a typical Xband spotlight SAR system capable of moderate resolution, the fractional bandwidth is of order $5 \%$.

The narrow aperture approximation (NAA) ignores reflectivity variation across the aperture. Ignoring dependence of the antenna gain function upon look direction, reflectivity dependence upon observation angle enters primarily through the local incidence angle. Variation in the cosine of local incidence is of order $1 \%$ for a typical X-band SAR. At near normal incidence, this corresponds to a change in local incidence of approximately 9 degrees, but only about 1 degree at 45 -degree incidence. Reflectivity variation also results from a change in local polarisation vectors across the aperture. Combining all reasonable estimates (but neglecting shadowing), the reflectivity function may vary by approximately $5 \%$ across the synthetic aperture used to form highresolution, X-band, spotlight SAR images. This magnitude of error is close to that expected for the NBA. Note that for highly conducting surfaces, such as those of man-made vehicles, reflection coefficients display little variation with incidence and can be assumed constant across the aperture.

Returning to the model for SAR pixels (15), the polarimetric pixel amplitude for the $p^{\text {th }}$ observation is

$$
\Omega_{\alpha \beta p}=\hat{\alpha}_{s} \cdot \underline{\underline{\Omega}}_{p} \cdot \hat{\beta}_{i}, \quad \alpha, \beta \in H, V .
$$

The definition of complex SAR coherence is

$$
\gamma_{\alpha \beta \gamma \delta}^{(p q)}=\frac{\left\langle\Omega_{\alpha \beta p} \Omega_{\gamma \delta q}^{*}\right\rangle}{\sqrt{\left\langle\Omega_{\alpha \beta p} \Omega_{\alpha \beta p}^{*}\right\rangle\left\langle\Omega_{\gamma \delta q} \Omega_{\gamma \delta q}^{*}\right\rangle}},
$$

where angular brackets indicate ensemble averaging. In practice, coherence estimates are obtained by averaging over a limited image region. Dropping polarimetric subscripts temporarily for the sake of clarity,

$$
\begin{aligned}
& \left\langle\Omega_{p} \Omega_{q}^{*}\right\rangle \\
& \quad=\iint_{S} \iint_{S} C_{p q}(\mathbf{r}, \mathbf{t}) e^{-i \kappa_{p}\left(\mathbf{r} \cdot \hat{y}_{p}\right)} e^{i \kappa_{q}\left(\mathbf{t} \cdot \hat{y}_{q}\right)} R_{p}(\mathbf{r}) R_{q}(\mathbf{t}) d \mathbf{r} d \mathbf{t},
\end{aligned}
$$

where subscripts denote observation dependent quantities and the surface reflectivity cross-correlation between observations $p$ and $q$ is

$$
\begin{aligned}
C_{p q}(\mathbf{r}, \mathbf{t})=\lim _{A \rightarrow \infty} \frac{1}{A} \iint_{A} & {\left[\Phi_{p}\left(\mathbf{r}^{\prime}+\mathbf{r}\right) e^{-i \kappa_{p} h_{p}\left(\mathbf{r}^{\prime}+\mathbf{r}\right) / \tan \theta_{p}}\right.} \\
& \left.\times \Phi_{q}^{*}\left(\mathbf{r}^{\prime}+\mathbf{t}\right) e^{i \kappa_{q} h_{q}\left(\mathbf{r}^{\prime}+\mathbf{t}\right) / \tan \theta_{q}}\right] d \mathbf{r}^{\prime} .
\end{aligned}
$$

Clearly, SAR coherence for mismatched apertures depends in general both upon the difference in system response function between observations and the cross-correlation in surface reflectivity. A short-ranged correlation $C_{p q}(\mathbf{r}, \mathbf{t})=C_{p q} \delta(\mathbf{r}-\mathbf{t})$ yields a coherency

$$
\gamma_{p q}=\frac{C_{p q}}{\sqrt{C_{p p} C_{q q}}} \frac{\iint e^{-i \kappa_{p}\left(\mathbf{r} \cdot \hat{y}_{p}\right)} e^{i \kappa_{q}\left(\mathbf{r} \cdot \hat{\mathbf{v}}_{q}\right)} R_{p}(\mathbf{r}) R_{q}(\mathbf{r}) d \mathbf{r}}{\sqrt{\iint R_{p}^{2}(\mathbf{r}) d \mathbf{r} \iint R_{q}^{2}(\mathbf{r}) d \mathbf{r}}}
$$

controlled by the mismatch in apertures as discussed in [10]. Alternatively, we may consider a surface reflectivity correlation long ranged in comparison with the SAR resolution. Under such circumstances, the correlation may be considered constant inside the integral of (18) such that

$$
\left\langle\Omega_{p} \Omega_{q}^{*}\right\rangle=C_{p q} \iint_{S} e^{-i \kappa_{p}\left(\mathbf{r} \cdot \hat{y}_{p}\right)} R_{p}(\mathbf{r}) d \mathbf{r} \iint_{S} e^{i \kappa_{q}\left(\mathbf{t} \cdot \hat{y}_{q}\right)} R_{q}(\mathbf{t}) d \mathbf{t}
$$

so that

$$
\begin{aligned}
\gamma_{p q}= & \frac{\left\langle\Omega_{p} \Omega_{q}^{*}\right\rangle}{\sqrt{\left\langle\Omega_{p} \Omega_{p}^{*}\right\rangle\left\langle\Omega_{q} \Omega_{q}^{*}\right\rangle}} \\
= & \frac{C_{p q}}{\sqrt{C_{p p} C_{q q}}} \\
& \times \frac{\iint e^{-i \kappa_{p}\left(\mathbf{r} \cdot \hat{y}_{p}\right)} R_{p}(\mathbf{r}) d \mathbf{r} \iint e^{-i \kappa_{q}\left(\mathbf{t} \cdot \hat{y}_{q}\right)} R_{q}(\mathbf{t}) d \mathbf{t}}{\sqrt{\left[\iint e^{-i \kappa_{p}\left(\mathbf{r} \cdot \hat{y}_{p}\right)} R_{p}(\mathbf{r}) d \mathbf{r}\right]^{2}\left[\iint e^{-i \kappa_{q}\left(\mathbf{t} \cdot \hat{y}_{q}\right)} R_{q}(\mathbf{t}) d \mathbf{t}\right]^{2}}} \\
= & \frac{C_{p q}}{\sqrt{C_{p p} C_{q q}}} .
\end{aligned}
$$

Such may be the case for highly conducting targets, and coherence may persist for such targets above that of natural terrain as aperture differences increase. Comparison of (20) with (22) reveals that coherence may vary depending upon surface reflectivity correlation properties, and although the significance of this effect is yet to be established, it is likely to have an increasing importance as SAR resolution improves. 


\section{SAR COHERENCY CALCULATIONS}

In order to calculate expected coherency due to scene changes, one might attempt to evaluate the correlations (19) for different types of change and combine with (17) and (18) to obtain coherence estimates. However, this approach yields only the mean coherence and not the higher moments of coherence: these latter may be defined using increasingly complex expressions, requiring higher-order reflectivity correlations.

In practice, the coherency between SAR images is estimated using a local average in a small window. Each window is centred on a different location and the scene changes are displaced with respect to each window. The practical calculation of coherency estimates naturally yields a distribution of estimated values as clutter varies throughout the region of change, even when the change itself is homogeneous.

Thus an alternative approach to coherence calculation is to mimic the actual process of estimation by returning simply to (15) and evaluating SAR pixel values for an ensemble of surfaces drawn from the same distribution. Pixel values may be calculated for regions of modelled surface prechange and postchange and used to estimate coherence in a manner completely analogous to actual practice. This approach has been adopted in this work.

Using Monte Carlo techniques, an ensemble of surfaces drawn from a specified distribution has been generated. For this work a Gaussian-correlated random surface was chosen with height standard deviation $\sigma$ of $0.0048 \mathrm{~m}$. At X-band, this corresponds to a wavenumber height standard deviation product $k \sigma$ of 1.0. The wavenumber correlation length product $k l$, defined in accordance with [5], is chosen as 6.02. These values place the surface within the limit of validity of the Kirchhoff model as determined by Thorsos and Jackson [14]. We note that the surface roughness, whilst possibly smaller than may be observed for natural rough surfaces, is appropriate for the type of artificial surface we wish to consider.

A periodic square area $0.81 \mathrm{~m}^{2}$ has been simulated in each instance, with a grid size of $0.003 \mathrm{~m}$. The simulation proceeds by estimating the dyadic reflectivity at each grid point on the surface, including the effects of geometrical shadowing. The reflectivity map has been used to evaluate the SAR pixel values by performing the integration (15a), but with a Hamming-weighted $k$-space sample [15] rather than the unweighted window of (15c).

For each surface pair used to estimate coherence, a grid of 9 SAR pixels has been used, at $3 \mathrm{~dB}$ azimuth and range resolutions of $0.15 \mathrm{~m}$; a pixel separation of $0.3 \mathrm{~m}$ was chosen to maximise pixel value independence, whilst maintaining computational efficiency, the centre wavelength was $0.03 \mathrm{~m}$ and soil dielectric permittivity was $15.7+j 4.7$.

Coherency estimates have been calculated using the 9pixel window to estimate the average in (17) for 1849 surface pair realisations. At an incidence angle of 39.7 degrees, soil surface backscattering coefficients were reported as $-12.4 \mathrm{~dB}$ $(\mathrm{HH}),-52.6 \mathrm{~dB}(\mathrm{HV})$, and $-12.2 \mathrm{~dB}(\mathrm{VV})$, in keeping with values reported in [5] and with observations of bare soil surfaces at X-band [16]. We note that the copolar surface brightness is orders of magnitude above the noise equivalent backscattering coefficient for a typical SAR.

In using the sample coherency as a scene change statistic, a simple threshold test is applied to discriminate between the areas in the scene subject to change and those that remain unchanged. In particular, values of the sample coherence greater than the threshold are considered to be realisations of the unchanged scene hypothesis, while values less than the threshold are considered to be realisations of the changed scene hypothesis. In order to assess the utility of the sample coherence as a change statistic, realistic values of the scene coherence under the changed and unchanged hypotheses need to be obtained so that the probability of false alarm and probability of detection may be derived as a function of the decision threshold.

In a numerical calculation, a repeat observation, with identical parameters and without change to the surface, will naturally yield a perfect coherence of one. In reality, a repeat observation will not yield a perfect coherence, and the observed coherence can be characterised as a product of several coherency factors,

$$
\gamma=\gamma_{\text {scene }} \gamma_{\text {snr }} \gamma_{\text {geom }} \gamma_{\text {reg }}
$$

corresponding to the coherency of the scene, and coherency reduction factors from the radar signal-to-noise ratio, collection geometry mismatch (i.e., interferometric baseline), and image registration. The loss of coherency due to variation in collection geometry is minimised by cropping the frequency domain components of the repeat-pass signal history data to a common aperture of support in the desired image formation plane [17]. Variations in $\gamma_{\text {snr }}$ may be minimised by flying accurate repeat-pass tracks, achieved using GPS navigation. The decorrelation due to the image registration process is dependent on the accuracy of the estimates of the image misregistration. The misregistration is estimated using a sample of 1024 pixels to estimate cross-correlation, quantised to $0.0625 \%$ of a resolution cell.

Over regions of the scene exhibiting modest coherency registration, correlation values $\gamma_{\text {reg }}$ in excess of 0.99 may be obtained [18]. In practice, however, differences between the repeat-pass flight tracks and spatial variations in the misregistration across the scene can degrade the registration accuracy. Given that $\gamma_{\text {snr }}, \gamma_{\text {geom }}$, and $\gamma_{\text {reg }}$ may be maintained constant or close to unity, the temporal variation in image coherency given in (23) will track the temporal variation in scene coherency $\gamma_{\text {scene, }}$, albeit at a lower level. In practice, this lower level is modelled as an equivalent coherency noise, and observationally this noise level is equivalent to approximately $7.2 \mathrm{~dB}$ below the $\mathrm{HH}$ backscattering coefficient [1]. Such a level of effective noise leads to a reduced coherency of

$$
\gamma=\gamma_{\text {scene }} \gamma_{\text {snr_eff }}=\gamma_{\text {scene }} \frac{1}{1+\left(\sigma_{n} / \sigma_{\mathrm{HH}}\right)}=0.84
$$

for the case of perfect scene coherence. This level of effective noise has been modelled in the calculations by adding 
TABLE 1: Estimated and underlying coherency values recovered from simulation. The three columns to the left indicate coherence by polarimetric channel for the case of no change in the surface, but an effective coherence "noise" of $-7.2 \mathrm{~dB}$ in the HH channel. The following columns indicate coherencies by polarimetric channel for changes corresponding to three types of tyre track: thin, medium, and wide, described in Table 2.

\begin{tabular}{lcccccccccccc}
\hline & \multicolumn{3}{c}{ No change } & \multicolumn{3}{c}{ Thin track } & \multicolumn{3}{c}{ Medium track } & \multicolumn{3}{c}{ Wide track } \\
& $\mathrm{HH}$ & $\mathrm{HV}$ & $\mathrm{VV}$ & $\mathrm{HH}$ & $\mathrm{HV}$ & $\mathrm{VV}$ & $\mathrm{HH}$ & $\mathrm{HV}$ & $\mathrm{VV}$ & $\mathrm{HH}$ & $\mathrm{HV}$ & $\mathrm{VV}$ \\
\hline Est. & 0.84 & 0.3 & 0.85 & 0.7 & 0.3 & 0.71 & 0.65 & 0.3 & 0.66 & 0.56 & 0.3 \\
ENL & 7 & 9 & 8 & 6 & 9 & 6 & 6 & 9 & 6 & 6 & 9 \\
True & 0.83 & 0.06 & 0.84 & 0.68 & 0.07 & 0.7 & 0.62 & 0.07 & 0.64 & 0.5 & 0.07 & 0.52 \\
\hline
\end{tabular}

random complex Gaussian scattering values to the calculated pixel values at the appropriate power level in both of the repeat-pass images prior to coherency estimation.

It is well known that the sample coherency evaluated over a finite window is a biased estimator of the true underlying scene coherency [19]. This bias is most evident at low coherency values. The numerical calculation of the sample coherence, however, yields a distribution of coherency estimates with a theoretical probability distribution function (PDF) of the form [20]

$$
p(\hat{\gamma})=2(N-1)\left(1-\gamma^{2}\right)^{N} \hat{\gamma}\left(1-\hat{\gamma}^{2}\right)^{(N-2)}{ }_{2} F_{1}\left(N, N ; 1 ; \gamma^{2} \hat{\gamma}^{2}\right),
$$

where ${ }_{2} F_{1}$ is the Gauss hypergeometric function. The PDF is specified by two parameters; an effective look number (ENL), $N$, indicating the number of independent samples in the window average, and the mean underlying coherency $\gamma$.

A number of techniques have been proposed for recovering suitably unbiased estimates of the underlying coherency and the ENL using the sample coherence estimates. These include a maximum a posteriori- (MAP-) based estimate originally proposed by Foster and Guinzy [21], and more recently, an estimate based on the statistics of the sample interferometric phase originally derived for the specific case of alongtrack interferometry has been proposed [22, 23]. In using the MAP-based technique, however, difficulties can arise in evaluating the hypergoemetric function in (25) especially for the case of high-coherence repeat-pass pairs.

On the other hand, the technique described in $[22,23]$ specifically requires the sample interferometric phase and modifications to the algorithm are needed to accommodate an across-track baseline that typically arises in repeat-pass interferometric imaging. In this paper, an alternative process of least-squares fitting of the known sample coherency PDF, given in (25), to the modelled simulated coherency distributions has been employed to recover suitably accurate estimates of the underlying coherency and the ENL.

Results for the initial calculation of the no-change scenario are reported in Table 1, and a graph of the distribution of simulated coherency with least-squares fitted curves appears in Figure 2.

This calculation creates pairs of SAR pixel values in two 3 by 3 windows. Each pixel has the same underlying scattering amplitude in each of the two observations, but each has a

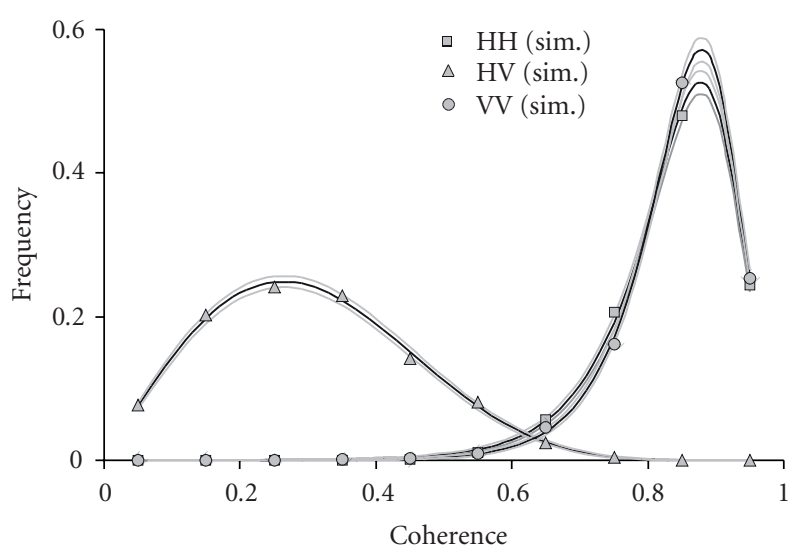

FIGURE 2: Simulated coherency distributions for the case of "no change" and the fitted theoretical distribution by polarimetric channel. Light-shaded curves indicate expected standard deviations in frequency based on a binomial random process for the number of samples in a histogram bin.

different realisation of the underlying noise. In fact, this calculation tells us little that could not be otherwise determined. However, it provides a useful test of the software used in later calculations and an illustration of the nature of the estimation process. Considering the $\mathrm{HH}$ channel first, the estimated coherence is 0.85 , indicating a slight bias against the true value of 0.84 . This value has been recovered by the process of fitting to the theoretical PDF, which also yields an ENL of 8 . This number is less than the actual number of pixels, 9 , as a consequence of the overlap in integration area for neighbouring pixels. There is some correlation between neighbouring pixel values due to this overlap, albeit rather small since pixel separations have been chosen at twice the width of the PSF at half the maximum height in power.

The greater backscattering coefficient of $\mathrm{VV}$ over $\mathrm{HH}$ leads to an improved signal-to-noise ratio in $\mathrm{VV}$ and hence a greater coherence in VV estimated at 0.86 , again indicating a slight bias over the true value of 0.85 . In the case of crosspolar returns, the $\mathrm{HV}$ or $\mathrm{VH}$ backscattering coefficient is some $27.9 \mathrm{~dB}$ below the HH backscattering coefficient. Thus when noise is added to the HV channel at the same level, it dominates the HV response. In this case, any correlation between neighbouring pixels is lost: the ENL is recovered as 9, and the biased estimated coherence is 0.3 , corresponding to a true coherence of zero, in line with the theoretical model 
TABle 2: Parameters describing the tyre track impressions made in the rough surfaces between simulated SAR observations. The edge distance is the thickness of the region over which the height and roughness return from their suppressed values to the unchanged values.

\begin{tabular}{lcccc}
\hline Track & Width $(\mathrm{m})$ & Depth $(\mathrm{m})$ & Edge $(\mathrm{m})$ & Roughness $/ \sigma$ \\
\hline Thin & 0.133 & 0.0067 & 0.0083 & 0.188 \\
Medium & 0.2 & 0.01 & 0.0125 & 0.125 \\
Wide & 0.3 & 0.015 & 0.0188 & 0.083 \\
\hline
\end{tabular}

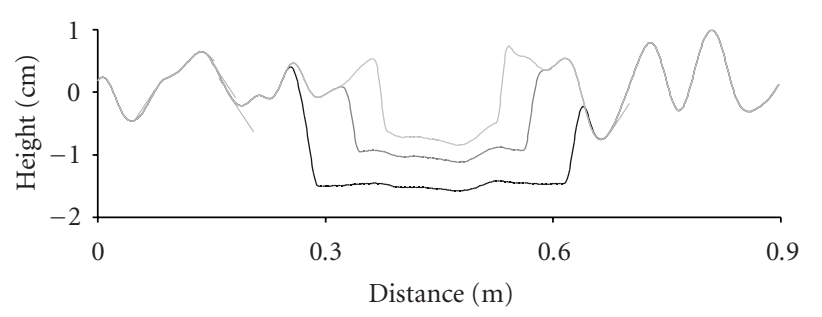

FIGURE 3: Height profiles recovered at a single range for a single realisation of the thin, medium, and wide tracks described in the text.

$[20,21,22]$. In practice, a cross-polar return at this level would be below the noise level of the radar and would not carry any information. We will not further consider crosspolar returns in this work.

Change due to tyre-track impressions has been modelled by creating a linear depression in each calculated surface. The depression properties are listed in Table 2. Three tracks have been modelled with different widths and depths; referred to as thin, medium, and wide tracks. Track changes have been modelled with arbitrary directions and displacements from the SAR pixel centres. In addition, the track change suppresses surface roughness within the track-changed area: the wide track reducing roughness the most and the thin track reducing roughness the least. Figure 3 is used to display sample height profiles across the track impression, whilst Figure 4 indicates the PSF-weighted surface reflectivities in the copolar channels for a single pixel in a single realisation of the track-changed surface.

It will be observed that changes to height within the track area, although appearing minor, yield a substantial change in phase in the returned signal. In addition, reflectivity variance within the track is reduced from that area of surface external to the track. These effects combine to influence the SAR pixel values, through (15), for pixels whose significant area of integration includes the track-changed area. This in turn alters the observed coherence, through (17), and the increasing reduction in coherence with increased change may be observed in Table 1 and in the plots of recovered and fitted coherency distributions displayed in Figure 5.

In the case of the $\mathrm{HH}$ polarisation, the underlying $\mathrm{co}^{-}$ herency changes from 0.84 in the case of no change to 0.68 for the thin track, 0.62 for the medium track, and 0.50 for the wide track. For VV, the higher coherency in the no-change scenario of 0.85 is reduced to 0.70 for the thin track, 0.64 for the medium track, and 0.52 for the wide track. In this

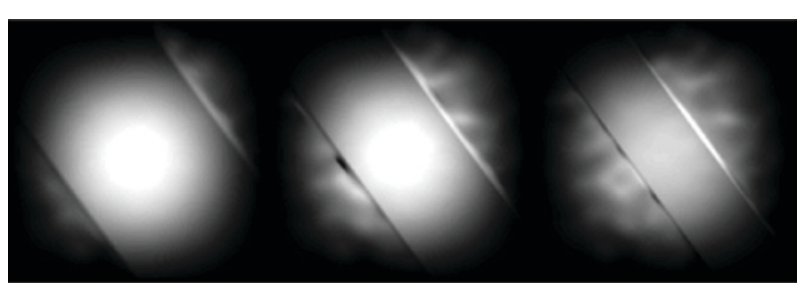

(a)

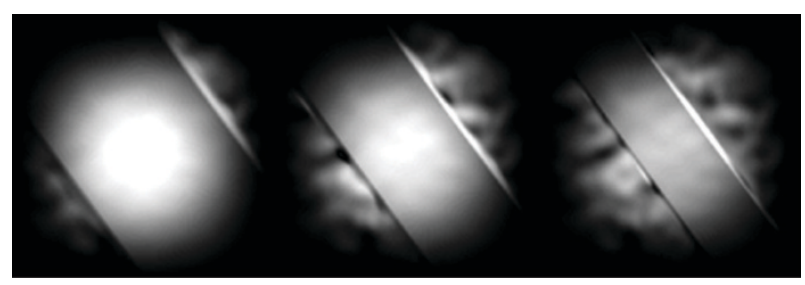

(b)

FIGURE 4: Graphic representation of (a) HH and (b) VV surface reflectivities modulated by the SAR point-spread function used to calculate SAR pixel values for a single pixel in a single realisation of the surface with the modelled tyre-track impression: wide track (on the left), medium track (centre), and thin track (on the right).

scenario, the coherency is almost linear in the track width, although we do not necessarily expect this relationship to persist for wider tracks.

There is clearly a marked difference between the distributions of coherence in the change and no-change cases that lends itself to exploitation in the detection of change. However, in the best case of the widest track, there remains a substantial overlap between the distributions that will tend to limit the utility of the coherency estimate for change detection. To quantify these ideas, the coherence distributions have been used to calculate receiver operator curves (ROCs) for the detection of the track changes using the copolar channels.

ROCs have been calculated in two ways: the first is by setting a threshold value of coherence and integrating the nochange coherency distribution to recover the probability of false alarm ( $\mathrm{P} f a$ ), whilst integrating the change coherency distribution to recover the probability of detection $(\mathrm{P} d)$. By sliding the threshold and plotting $\mathrm{P} d$ against $\mathrm{P} f a$, the ROC is determined. ROCs calculated in this way are displayed in Figure 6. 


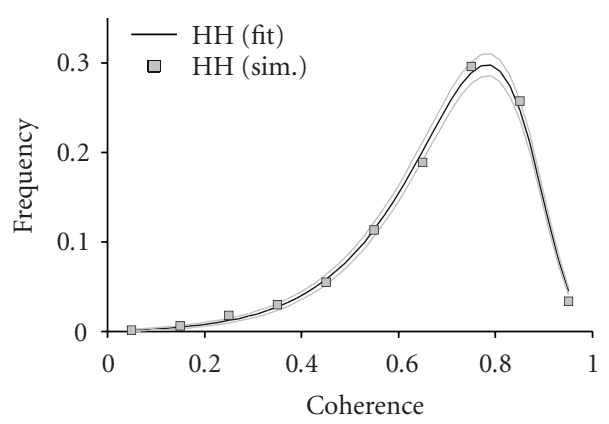

(a)

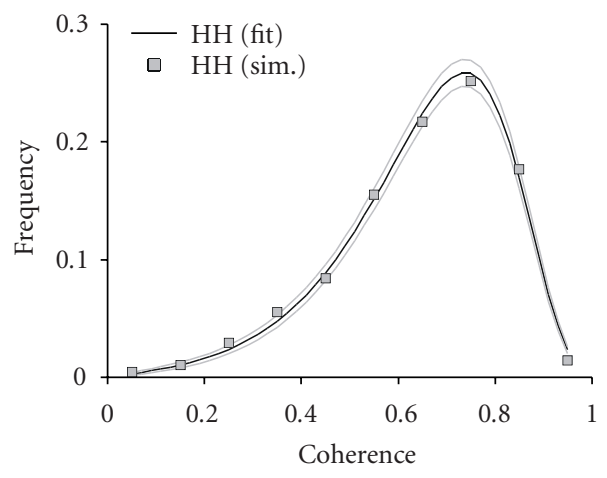

(c)

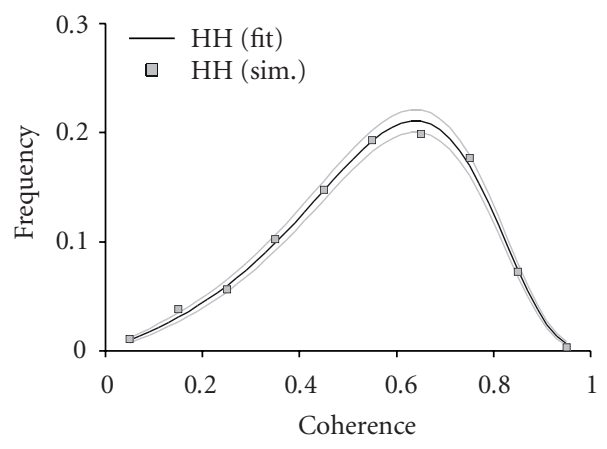

(e)

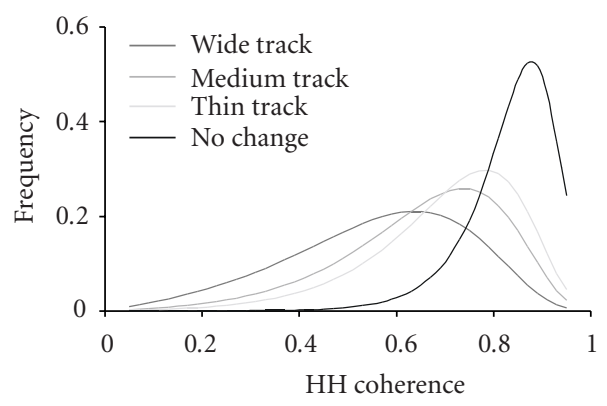

(g)

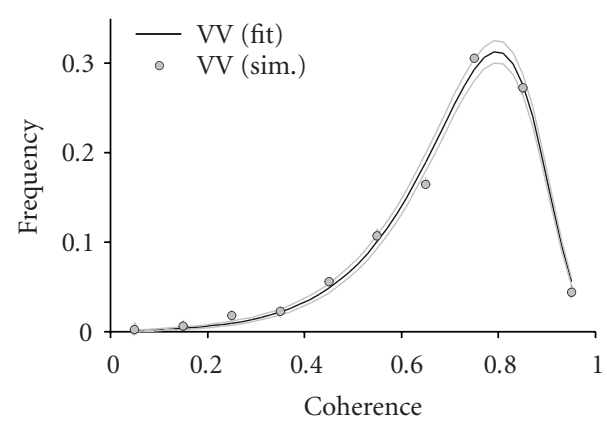

(b)

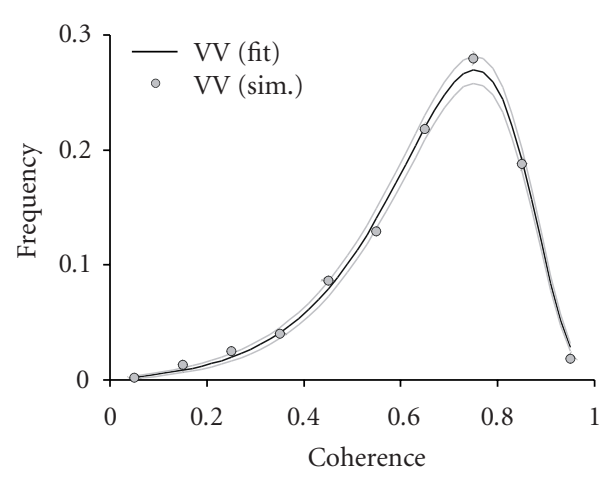

(d)

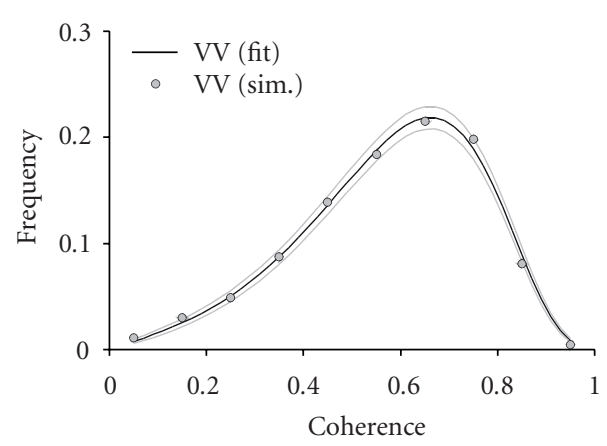

(f)

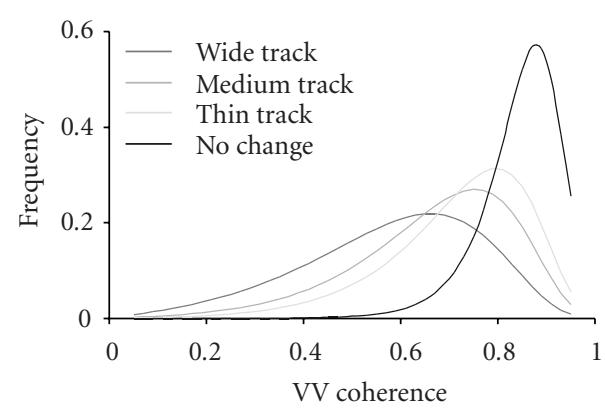

(h)

Figure 5: Coherency estimate histograms for (a), (b) thin track, (c), (d) medium track, and (e), (f) wide track. HH on the left and VV on the right. $(\mathrm{g})$, (h) The three fitted curves for each polarisation along with the distribution for the case of no change. In the first three rows, the light curves above and below the dark curve indicate the expected standard deviation. 


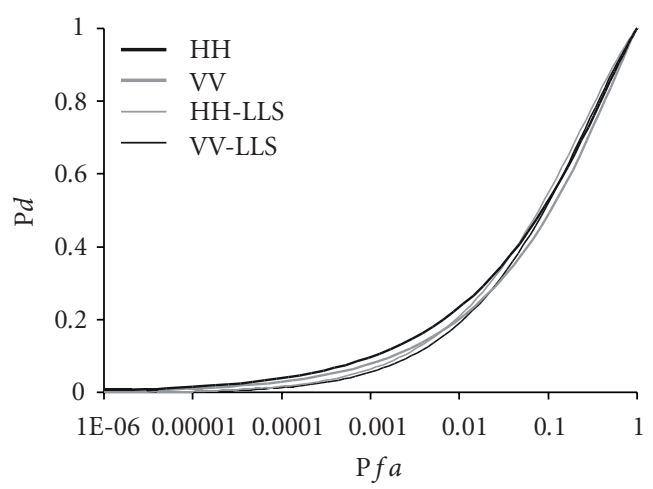

(a)

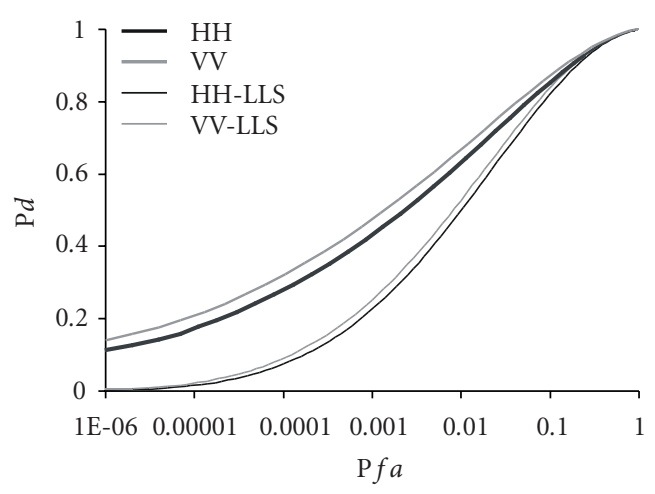

(c)

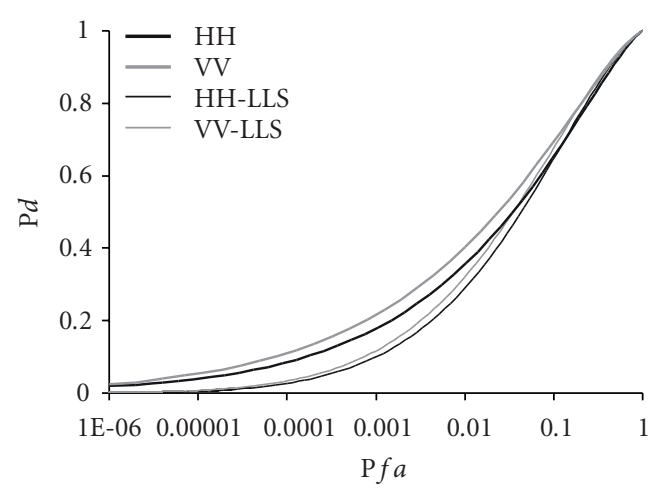

(b)

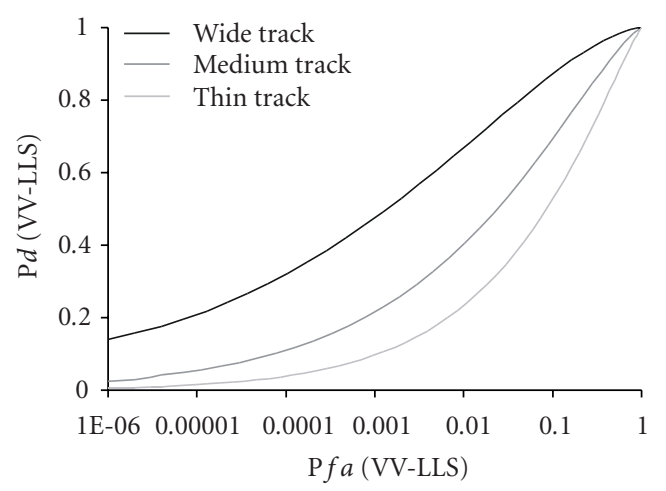

(d)

FIGURE 6: ROC curves determined using the coherency distributions recovered for the simulated copolar channels. (a) Thin track and (b) medium track. (c) Wide track and (d) all tracks in the VV, log-likelihood case.

Figure 6 also shows theoretical ROC curves for a loglikelihood change statistic $[1,24,25]$ that may be evaluated for the case when the full 2 by 2 sample covariance matrix describing the interferometric image pair is available for discriminating between the changed and unchanged scenarios. The log-likelihood change statistic is based on the assumption that the $N$-pixel region under test in the repeat-pass image pair is described by a complex, circular, jointly Gaussian random process. It is obtained by forming the likelihood ratio defined as the ratio of the probability that the $N$ pixel pairs in the local area under test are realisations of the unchanged hypothesis to the probability that the $N$ pixel pairs are realisations of the changed hypothesis [24].

In order to specify the probability distributions in the log-likelihood change, statistic knowledge of the full covariance matrices describing the changed and unchanged hypotheses is required. In practice, these are unknown and need to be estimated from the data in combination with judicious use of a priori information and assumptions regarding the nature of the scene and the anticipated scene changes [25]. In evaluating the theoretical ROC curves of Figure 6, estimates of the changed and unchanged scene covariance matrices have been formulated using the underlying coherence values obtained from the least-squares fitting process. It has also been assumed that the mean backscatter power of the scene is the same in both scenarios and that the interferometric phase remains the same. Although these assumptions are not strictly true (the mean backscatter power is a function of the surface roughness and the interferometric phase varies with the terrain topography), they are reasonable for the modest scene changes considered here and would typically be made in practice in the absence of other a priori information. The log-likelihood statistic (LLS) ROC curves of Figure 6 thus show the theoretical performance that may be obtained using a 3 by 3 spatial estimation pixel window applied to the simulated changed and unchanged scenes. The improvements in detection performance may be attributed to the use of the complete 2 by 2 covariance matrix describing the repeat-pass image pair to discriminate between the changed and unchanged hypotheses [26].

As noted the ROCs reveal that using VV polarisation to detect the changes in this rough-surface type yields better performance than using $\mathrm{HH}$. This result will depend upon the nature of the surface, wavelength, and imaging geometry. However, since VV surface backscatter is generally brighter than $\mathrm{HH}$, the result will often hold true. The improvement is most noticeable for the wider tracks and for the log-likelihood statistic calculation. 
TABLe 3: Probability of false alarm for a probability of detection of 1 in 10 for the different track changes, polarisation channels, and detection techniques.

\begin{tabular}{llccr}
\hline Track & $\mathrm{P} f a(\mathrm{HH})$ & $\mathrm{P} f a(\mathrm{VV})$ & $\mathrm{P} f a(\mathrm{HH}-\mathrm{LLS})$ & $\mathrm{P} f a(\mathrm{VV}-\mathrm{LLS})$ \\
\hline Thin & $2.9 \times 10^{-3}$ & $2.5 \times 10^{-3}$ & $1.6 \times 10^{-3}$ & $1.1 \times 10^{-3}$ \\
Medium & $1.0 \times 10^{-3}$ & $7.4 \times 10^{-4}$ & $1.8 \times 10^{-4}$ & $7.9 \times 10^{-5}$ \\
Wide & $1.8 \times 10^{-4}$ & $1.2 \times 10^{-4}$ & $<1.0 \times 10^{-6}$ & $<1.0 \times 10^{-6}$ \\
\hline
\end{tabular}

To demonstrate the utility of SAR CCD for this scenario, we tabulate the probability of false alarm against change type in Table 3, where false-alarm rates are reported for a probability of detection of 1 in 10 . This detection rate may appear low, but tracks are extended features in an image and their detection does not rely on the detection of each and every pixel in the track: rather the human operator will naturally correlate detections distributed along the line of track, even though the track appears incomplete.

From Table 3, we see that for the thin track, with width slightly less than a resolution length, the false-alarm rate is high at approximately $1 / 400$. The medium track has a width $33 \%$ greater than the SAR resolution and the false-alarm rates for the 1 in 10 detection rate are lower at around 1/1000, depending upon polarisation. The log-likelihood method yields an improvement to between $1 / 6000$ and $1 / 12000$. Finally, the wide track, with a width double that of the SAR resolution, yields the lowest false-alarm rates of approximately $1 / 8000$ for the straightforward ROC calculation and less than 1/1000 000 for the log-likelihood statistic. Thus we expect that, in the absence of other changes, tracks of this dimension will be reliably detected by such a SAR system.

To justify this last statement, we compare in Figure 7 the calculated PDFs for the VV case and the three track sizes against that observed for real-tyre changes in a rough surface [27]. The reader is referred to [27] for the detailed discussion of the observation. The observed coherence PDF lies very close to that of our medium track calculation and corresponds to an underlying coherence of 6.3 and an ENL of 5 . The background coherence distribution for the rough surface corresponds precisely to the case of no change modelled here. The observed coherence values from which this distribution has been obtained are also illustrated in the image of Figure 7 . Tyre tracks in this coherence image are clearly visible to the eye. Thus we conclude with confidence that our medium-to-wide tyre tracks will be detected by the modelled SAR CCD system under the prevailing conditions of natural change between observations.

This last statement contains the most important phrase for what we have not considered here: the length of the time interval between observation, and the natural effects of weathering upon scene coherence. Recent evidence [4] suggests that coherence at the unchanged level supposed in this calculation, and in the observation, may persist for several hours, and under benign conditions may remain sufficiently high for several days. Thus, our inference concerning the ability to detect change may be valid at least for several hours.

\section{CONCLUSIONS}

We have combined a physical model for surface scattering with a signal processing model for spotlight SAR observation to derive an expression for SAR coherence from random rough surfaces. Numerical calculations conducted within the context of the model have been used to predict the performance of a high-resolution, X-band, repeat-pass, spotlight SAR CCD system. In accordance with observations, ROC curves calculated using a log-likelihood statistic indicate that such a system may be used to detect changes associated with tyre tracks resulting from vehicle passage when those tracks are wider than the SAR resolution.

\section{APPENDICES}

\section{A. DYADIC SURFACE BACKSCATTER REFLECTIVITY FOR A DIELECTRIC SURFACE IN THE TANGENT PLANE APPROXIMATION}

Given the relationship

$$
\underline{\underline{F}} \cdot \mathbf{E}_{i}=\frac{i k}{4 \pi}\left[\underline{\underline{I}}-\hat{\mathbf{k}}_{s} \hat{\mathbf{k}}_{s}\right] \cdot\left\{\eta[\hat{\mathbf{n}} \times \mathbf{H}]+\left[\hat{\mathbf{k}}_{s} \times \hat{\mathbf{n}} \times \mathbf{E}\right]\right\}
$$

and the surface tangential fields in the Kirchhoff approximation $[5,6,7]$

$$
\begin{array}{r}
\hat{\mathbf{n}} \times \mathbf{E}=\left\{\left(1+R_{h}\right)\left(\hat{\mathbf{n}} \times \widehat{\mathbf{h}}_{i}\right) \hat{\mathbf{h}}_{i}-\left(1-R_{v}\right)\left(\hat{\mathbf{n}} \cdot \hat{\mathbf{k}}_{i}\right) \hat{\mathbf{h}}_{i} \hat{\mathbf{v}}_{i}\right\} \cdot \mathbf{E}_{i}, \\
(\mathrm{~A} .2) \\
-\eta[\hat{\mathbf{n}} \times \mathbf{H}]=\left\{\left(1+R_{v}\right)\left(\hat{\mathbf{n}} \times \widehat{\mathbf{h}}_{i}\right) \widehat{\mathbf{v}}_{i}+\left(1-R_{h}\right)\left(\widehat{\mathbf{n}} \cdot \hat{\mathbf{k}}_{i}\right) \widehat{\mathbf{h}}_{i} \hat{\mathbf{h}}_{i}\right\} \cdot \mathbf{E}_{i},
\end{array}
$$

we seek to show that

$$
\underline{\underline{F}}=\frac{i k}{2 \pi}\left(\widehat{\mathbf{n}} \cdot \widehat{\mathbf{k}}_{i}\right)\left[R_{h} \widehat{\mathbf{h}}_{i} \widehat{\mathbf{h}}_{i}-R_{v} \widehat{\mathbf{v}}_{i} \widehat{\mathbf{v}}_{i}\right]
$$

Define the local polarisation vectors for the incident and reflected waves using the local surface outward normal vector $\widehat{\mathbf{n}}[6]$ :

$$
\begin{aligned}
\hat{\mathbf{h}}_{i}=\frac{\hat{\mathbf{k}}_{i} \times \hat{\mathbf{n}}}{\left|\hat{\mathbf{k}}_{i} \times \hat{\mathbf{n}}\right|}, & \hat{\mathbf{h}}_{r}=\frac{\hat{\mathbf{k}}_{r} \times \hat{\mathbf{n}}}{\left|\hat{\mathbf{k}}_{r} \times \hat{\mathbf{n}}\right|}, \\
\hat{\mathbf{v}}_{i}=\hat{\mathbf{k}}_{i} \times \hat{\mathbf{h}}_{i}, & \hat{\mathbf{v}}_{r}=\hat{\mathbf{k}}_{r} \times \hat{\mathbf{h}}_{r} .
\end{aligned}
$$




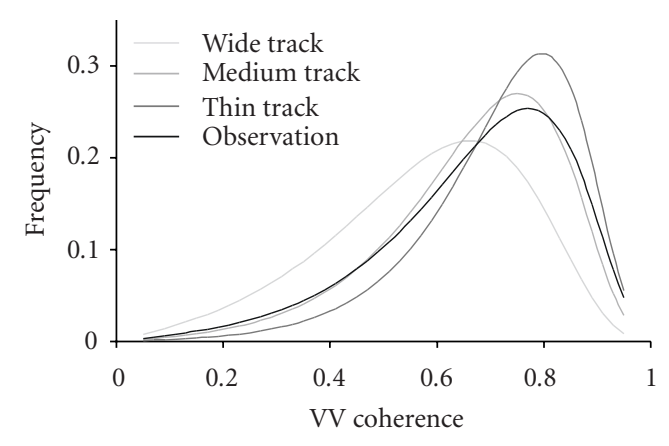

(a)

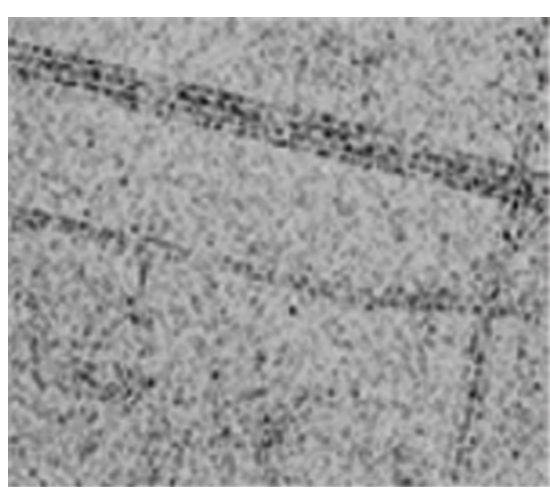

(b)

Figure 7: (a) Comparison of simulated coherence PDFs with that observed for the passage of a vehicle over a surface as reported in [27]. The coherence image from which the observed distribution has been obtained is shown in (b), wherein tyre tracks are clearly discernible by eye.

Since

$$
\widehat{\mathbf{k}}_{r}=\widehat{\mathbf{k}}_{i}-2 \widehat{\mathbf{n}}\left(\widehat{\mathbf{k}}_{i} \cdot \widehat{\mathbf{n}}\right)
$$

we have

$\widehat{\mathbf{h}}_{r}=\frac{\hat{\mathbf{k}}_{r} \times \hat{\mathbf{n}}}{\left|\hat{\mathbf{k}}_{r} \times \hat{\mathbf{n}}\right|}=\frac{1}{\left|\hat{\mathbf{k}}_{r} \times \hat{\mathbf{n}}\right|}\left(\widehat{\mathbf{k}}_{i}-2 \widehat{\mathbf{n}}\left(\hat{\mathbf{k}}_{i} \cdot \hat{\mathbf{n}}\right)\right) \times \hat{\mathbf{n}}=\frac{\hat{\mathbf{k}}_{i} \times \hat{\mathbf{n}}}{\left|\hat{\mathbf{k}}_{i} \times \hat{\mathbf{n}}\right|}=\widehat{\mathbf{h}}_{i}$,

$\widehat{\mathbf{v}}_{r}=\widehat{\mathbf{k}}_{r} \times \widehat{\mathbf{h}}_{r}=\left(\hat{\mathbf{k}}_{i}-2 \widehat{\mathbf{n}}\left(\hat{\mathbf{k}}_{i} \cdot \hat{\mathbf{n}}\right)\right) \times \widehat{\mathbf{h}}_{i}=\widehat{\mathbf{v}}_{i}-2\left(\hat{\mathbf{k}}_{i} \cdot \hat{\mathbf{n}}\right) \hat{\mathbf{n}} \times \widehat{\mathbf{h}}_{i}$.

The incident electric field may be written as

$$
\mathbf{E}_{i}=E_{o} \hat{\mathbf{a}}=E_{o}\left\{\left(\hat{\mathbf{a}} \cdot \widehat{\mathbf{h}}_{i}\right) \widehat{\mathbf{h}}_{i}+\left(\hat{\mathbf{a}} \cdot \widehat{\mathbf{v}}_{i}\right) \widehat{\mathbf{v}}_{i}\right\}
$$

In order to express the reflectivity in terms of the surface fields, define a local Fresnel reflection dyadic

$$
\underline{\underline{R}}=R_{h} \widehat{\mathbf{h}}_{r} \widehat{\mathbf{h}}_{i}+R_{v} \widehat{\mathbf{v}}_{r} \widehat{\mathbf{v}}_{i}
$$

where $R_{h}$ and $R_{v}$ are the local Fresnel reflection coefficients. In the tangent plane approximation, the total field at a point on the surface is the sum of the incident field and the field reflected from an infinite plane tangent at that point, that is,

$$
\mathbf{E}=\mathbf{E}_{i}+\mathbf{E}_{r}=[\underline{\underline{I}}+\underline{\underline{R}}] \cdot \mathbf{E}_{i}
$$

To derive (A.4), we may work directly from (A.2); to use (A.3), we must first show that it can be written using (A.11). We start by noting that for the harmonic wave,

$$
\begin{gathered}
i k \eta \mathbf{H}_{i}=i \mathbf{k}_{i} \times \mathbf{E}_{i}=i k \hat{\mathbf{k}}_{i} \times \mathbf{E}_{i}, \\
i k \eta \mathbf{H}_{r}=i \mathbf{k}_{r} \times \mathbf{E}_{r}=i k \hat{\mathbf{k}}_{r} \times\left(\underline{\underline{R}} \cdot \mathbf{E}_{i}\right),
\end{gathered}
$$

so that

$$
\begin{aligned}
& \eta[\hat{\mathbf{n}} \times \mathbf{H}] \\
& =\eta\left[\hat{\mathbf{n}} \times\left(\mathbf{H}_{i}+\mathbf{H}_{r}\right)\right] \\
& =\widehat{\mathbf{n}} \times \widehat{\mathbf{k}}_{i} \times \mathbf{E}_{i}+\hat{\mathbf{n}} \times \widehat{\mathbf{k}}_{r} \times\left(\underline{\underline{R}} \cdot \mathbf{E}_{i}\right) \\
& =\hat{\mathbf{k}}_{i}\left(\hat{\mathbf{n}} \cdot \mathbf{E}_{i}\right)-\mathbf{E}_{i}\left(\hat{\mathbf{n}} \cdot \hat{\mathbf{k}}_{i}\right)+\widehat{\mathbf{n}} \times \widehat{\mathbf{k}}_{r} \times\left(\underline{\underline{R}} \cdot \mathbf{E}_{i}\right) \\
& =\left[\left(\hat{\mathbf{k}}_{i} \hat{\mathbf{n}}\right)-\left(\hat{\mathbf{n}} \cdot \hat{\mathbf{k}}_{i}\right) \underline{\underline{I}}\right] \cdot \mathbf{E}_{i}+\left[\left(\hat{\mathbf{k}}_{r} \widehat{\mathbf{n}}\right)-\left(\hat{\mathbf{n}} \cdot \hat{\mathbf{k}}_{r}\right) \underline{\underline{I}}\right] \cdot \underline{\underline{R}} \cdot \mathbf{E}_{i} \\
& =\left[\left(\hat{\mathbf{k}}_{i} \hat{\mathbf{n}}\right)-\left(\widehat{\mathbf{n}} \cdot \hat{\mathbf{k}}_{i}\right) \underline{\underline{I}}+\left(\hat{\mathbf{k}}_{r} \hat{\mathbf{n}}\right) \cdot \underline{\underline{R}}-\left(\hat{\mathbf{n}} \cdot \hat{\mathbf{k}}_{r}\right) \underline{\underline{R}}\right] \cdot \mathbf{E}_{i} .
\end{aligned}
$$

To show equivalence between (A.13) and (A.3), we substitute for the incident field in the second line of (A.13) using (A.9):

$$
\begin{aligned}
& \eta[\hat{\mathbf{n}} \times \mathbf{H}]=\hat{\mathbf{n}} \times \hat{\mathbf{k}}_{i} \times \mathbf{E}_{i}+\widehat{\mathbf{n}} \times \widehat{\mathbf{k}}_{r} \times\left(\underline{\underline{R}} \cdot \mathbf{E}_{i}\right) \\
& =\widehat{\mathbf{n}} \times \widehat{\mathbf{k}}_{i} \times\left(\left(\hat{\mathbf{a}} \cdot \hat{\mathbf{h}}_{i}\right) \hat{\mathbf{h}}_{i}+\left(\hat{\mathbf{a}} \cdot \hat{\mathbf{v}}_{i}\right) \widehat{\mathbf{v}}_{i}\right)+\widehat{\mathbf{n}} \times \widehat{\mathbf{k}}_{r} \\
& \times\left(\left(R_{h} \hat{\mathbf{h}}_{r} \hat{\mathbf{h}}_{i}+R_{v} \widehat{\mathbf{v}}_{r} \widehat{\mathbf{v}}_{i}\right) \cdot\left(\left(\hat{\mathbf{a}} \cdot \widehat{\mathbf{h}}_{i}\right) \hat{\mathbf{h}}_{i}+\left(\hat{\mathbf{a}} \cdot \widehat{\mathbf{v}}_{i}\right) \widehat{\mathbf{v}}_{i}\right)\right) \\
& =\left(\hat{\mathbf{a}} \cdot \widehat{\mathbf{h}}_{i}\right)\left(\hat{\mathbf{n}} \times \hat{\mathbf{k}}_{i} \times \widehat{\mathbf{h}}_{i}\right)+\left(\hat{\mathbf{a}} \cdot \hat{\mathbf{v}}_{i}\right)\left(\hat{\mathbf{n}} \times \widehat{\mathbf{k}}_{i} \times \widehat{\mathbf{v}}_{i}\right) \\
& +R_{h}\left(\hat{\mathbf{a}} \cdot \hat{\mathbf{h}}_{i}\right)\left(\hat{\mathbf{n}} \times \hat{\mathbf{k}}_{r} \times \widehat{\mathbf{h}}_{r}\right) \\
& +R_{v}\left(\hat{\mathbf{a}} \cdot \widehat{\mathbf{v}}_{i}\right)\left(\hat{\mathbf{n}} \times \widehat{\mathbf{k}}_{r} \times \widehat{\mathbf{v}}_{r}\right) .
\end{aligned}
$$

Now

$$
\widehat{\mathbf{n}} \times \widehat{\mathbf{k}}_{i} \times \widehat{\mathbf{h}}_{i}=\widehat{\mathbf{k}}_{i}\left(\widehat{\mathbf{n}} \cdot \widehat{\mathbf{h}}_{i}\right)-\widehat{\mathbf{h}}_{i}\left(\widehat{\mathbf{n}} \cdot \widehat{\mathbf{k}}_{i}\right)=-\widehat{\mathbf{h}}_{i}\left(\widehat{\mathbf{n}} \cdot \widehat{\mathbf{k}}_{i}\right)
$$


Using this result in (A.14) yields

$$
\begin{aligned}
& \eta[\hat{\mathbf{n}} \times \mathbf{H}] \\
& =-\widehat{\mathbf{h}}_{i}\left(\hat{\mathbf{a}} \cdot \hat{\mathbf{h}}_{i}\right)\left(\left(\hat{\mathbf{n}} \cdot \hat{\mathbf{k}}_{i}\right)+R_{h}\left(\hat{\mathbf{n}} \cdot \widehat{\mathbf{k}}_{r}\right)\right) \\
& +\left(\hat{\mathbf{a}} \cdot \hat{\mathbf{v}}_{i}\right)\left(\hat{\mathbf{n}} \times \hat{\mathbf{k}}_{i} \times \widehat{\mathbf{v}}_{i}\right)+R_{v}\left(\hat{\mathbf{a}} \cdot \widehat{\mathbf{v}}_{i}\right)\left(\hat{\mathbf{n}} \times \hat{\mathbf{k}}_{r} \times \widehat{\mathbf{v}}_{r}\right) \\
& =-\hat{\mathbf{h}}_{i}\left(\hat{\mathbf{a}} \cdot \hat{\mathbf{h}}_{i}\right)\left(\hat{\mathbf{n}} \cdot \hat{\mathbf{k}}_{i}\right)\left(1-R_{h}\right) \\
& +\left(\hat{\mathbf{a}} \cdot \hat{\mathbf{v}}_{i}\right)\left(\hat{\mathbf{n}} \times \hat{\mathbf{k}}_{i} \times \widehat{\mathbf{v}}_{i}\right)+R_{v}\left(\hat{\mathbf{a}} \cdot \hat{\mathbf{v}}_{i}\right)\left(\hat{\mathbf{n}} \times \hat{\mathbf{k}}_{r} \times \widehat{\mathbf{v}}_{r}\right) .
\end{aligned}
$$

Finally, noting that

$$
\begin{aligned}
\left(\hat{\mathbf{n}} \times \hat{\mathbf{k}}_{i} \times \hat{\mathbf{v}}_{i}\right) & =\hat{\mathbf{n}} \times\left(\hat{\mathbf{k}}_{i} \times \hat{\mathbf{k}}_{i} \times \hat{\mathbf{h}}_{i}\right) \\
& =\hat{\mathbf{n}} \times\left(\hat{\mathbf{k}}_{i}\left(\hat{\mathbf{k}}_{i} \cdot \hat{\mathbf{h}}_{i}\right)-\hat{\mathbf{h}}_{i}\left(\hat{\mathbf{k}}_{i} \cdot \hat{\mathbf{k}}_{i}\right)\right) \\
& =-\hat{\mathbf{n}} \times \hat{\mathbf{h}}_{i},
\end{aligned}
$$

it is clear that (A.16) can be written as

$$
\begin{aligned}
\eta[\hat{\mathbf{n}} \times \mathbf{H}]= & -\left(1-R_{h}\right)\left(\hat{\mathbf{n}} \cdot \hat{\mathbf{k}}_{i}\right)\left(\hat{\mathbf{a}} \cdot \hat{\mathbf{h}}_{i}\right) \hat{\mathbf{h}}_{i} \\
& -\left(1+R_{v}\right)\left(\hat{\mathbf{a}} \cdot \widehat{\mathbf{v}}_{i}\right)\left(\widehat{\mathbf{n}} \times \widehat{\mathbf{h}}_{i}\right) .
\end{aligned}
$$

Thus, (A.13) is equivalent to (A.3) and we may use (A.13) in place of (A.3) in the derivation of the reflection dyadic. In fact, it is readily shown that (A.11) may be used to derive the tangential electric field (A.2) in a similar fashion. From (A.2), it is clear that

$$
\begin{aligned}
\hat{\mathbf{k}}_{s} \times \hat{\mathbf{n}} \times \mathbf{E} & \\
= & {\left[\left(1+R_{h}\right)\left(\hat{\mathbf{k}}_{s} \times \hat{\mathbf{n}} \times \widehat{\mathbf{h}}_{i}\right) \hat{\mathbf{h}}_{i}\right.} \\
& \left.\quad-\left(1-R_{v}\right)\left(\hat{\mathbf{n}} \cdot \hat{\mathbf{k}}_{i}\right)\left(\hat{\mathbf{k}}_{s} \times \widehat{\mathbf{h}}_{i}\right) \widehat{\mathbf{v}}_{i}\right] \cdot \mathbf{E}_{i} \\
= & \underline{\underline{F}}_{E} \cdot \mathbf{E}_{i},
\end{aligned}
$$

and from (A.13), we have

$$
\begin{aligned}
\eta[\hat{\mathbf{n}} \times \mathbf{H}] & =\left[\left(\hat{\mathbf{k}}_{i} \hat{\mathbf{n}}\right)-\left(\hat{\mathbf{n}} \cdot \hat{\mathbf{k}}_{i}\right) \underline{\underline{I}}+\left(\hat{\mathbf{k}}_{r} \hat{\mathbf{n}}\right) \cdot \underline{\underline{R}}-\left(\hat{\mathbf{n}} \cdot \hat{\mathbf{k}}_{r}\right) \underline{\underline{R}}\right] \cdot \mathbf{E}_{i} \\
& =\underline{\underline{F}}_{H} \cdot \mathbf{E}_{i} .
\end{aligned}
$$

Thus the reflectivity density in the form sought is

$$
\begin{aligned}
\underline{\underline{F}}=\frac{i k}{4 \pi}\left[\underline{\underline{I}}-\hat{\mathbf{k}}_{s} \hat{\mathbf{k}}_{s}\right] \cdot\left\{\underline{\underline{F}}_{E}+\underline{\underline{F}}_{H}\right\} \\
=\frac{i k}{4 \pi}\left[\underline{\underline{I}}-\widehat{\mathbf{k}}_{s} \hat{\mathbf{k}}_{s}\right] \cdot\left[\left(1+R_{h}\right)\left(\hat{\mathbf{k}}_{s} \times \hat{\mathbf{n}} \times \hat{\mathbf{h}}_{i}\right) \hat{\mathbf{h}}_{i}\right. \\
\left.-\left(1-R_{v}\right)\left(\hat{\mathbf{n}} \cdot \hat{\mathbf{k}}_{i}\right)\left(\hat{\mathbf{k}}_{s} \times \widehat{\mathbf{h}}_{i}\right) \hat{\mathbf{v}}_{i}\right] \\
+\frac{i k}{4 \pi}\left[\underline{\underline{I}}-\hat{\mathbf{k}}_{s} \hat{\mathbf{k}}_{s}\right] \cdot\left[\left(\hat{\mathbf{k}}_{i} \hat{\mathbf{n}}\right)-\left(\hat{\mathbf{n}} \cdot \hat{\mathbf{k}}_{i}\right) \underline{\underline{I}}+\left(\hat{\mathbf{k}}_{r} \hat{\mathbf{n}}\right) \cdot \underline{\underline{R}}\right. \\
\left.-\left(\hat{\mathbf{n}} \cdot \hat{\mathbf{k}}_{r}\right) \underline{\underline{R}}\right] .
\end{aligned}
$$

To simplify further, note that for backscatter,

$$
\begin{aligned}
\left(\hat{\mathbf{k}}_{s} \times \widehat{\mathbf{n}} \times \widehat{\mathbf{h}}_{i}\right) & =\widehat{\mathbf{h}}_{i}\left(\hat{\mathbf{n}} \cdot \hat{\mathbf{k}}_{i}\right), \\
\left(\hat{\mathbf{k}}_{s} \times \widehat{\mathbf{h}}_{i}\right) & =-\widehat{\mathbf{v}}_{i},
\end{aligned}
$$

so that

$$
\begin{aligned}
\underline{\underline{F}=}= & \frac{i k}{4 \pi}\left(\hat{\mathbf{n}} \cdot \hat{\mathbf{k}}_{i}\right)\left[\left(1+R_{h}\right) \hat{\mathbf{h}}_{i} \hat{\mathbf{h}}_{i}+\left(1-R_{v}\right) \hat{\mathbf{v}}_{i} \hat{\mathbf{v}}_{i}\right] \\
& +\frac{i k}{4 \pi}\left[\underline{\underline{I}}-\hat{\mathbf{k}}_{i} \hat{\mathbf{k}}_{i}\right] \\
& \cdot\left[\left(\hat{\mathbf{k}}_{i} \hat{\mathbf{n}}\right)-\left(\widehat{\mathbf{n}} \cdot \hat{\mathbf{k}}_{i}\right) \underline{\underline{I}}+\left(\hat{\mathbf{k}}_{r} \hat{\mathbf{n}}\right) \cdot \underline{\underline{R}}-\left(\hat{\mathbf{n}} \cdot \hat{\mathbf{k}}_{r}\right) \underline{\underline{R}}\right] .
\end{aligned}
$$

Expanding inside the final term,

$$
\begin{aligned}
& {\left[\underline{\underline{I}}-\hat{\mathbf{k}}_{i} \hat{\mathbf{k}}_{i}\right] \cdot\left[\left(\hat{\mathbf{k}}_{i} \hat{\mathbf{n}}\right)-\left(\hat{\mathbf{n}} \cdot \hat{\mathbf{k}}_{i}\right) \underline{\underline{I}}\right]} \\
& =\left[\left(\hat{\mathbf{k}}_{i} \hat{\mathbf{n}}\right)-\left(\widehat{\mathbf{n}} \cdot \hat{\mathbf{k}}_{i}\right) \underline{\underline{I}}-\left(\hat{\mathbf{k}}_{i} \hat{\mathbf{n}}\right)+\left(\hat{\mathbf{n}} \cdot \hat{\mathbf{k}}_{i}\right) \hat{\mathbf{k}}_{i} \hat{\mathbf{k}}_{i}\right] \\
& =-\left(\hat{\mathbf{n}} \cdot \hat{\mathbf{k}}_{i}\right)\left[\underline{\underline{I}}-\hat{\mathbf{k}}_{i} \hat{\mathbf{k}}_{i}\right] \\
& =-\left(\hat{\mathbf{n}} \cdot \hat{\mathbf{k}}_{i}\right)\left[\hat{\mathbf{h}}_{i} \hat{\mathbf{h}}_{i}+\widehat{\mathbf{v}}_{i} \widehat{\mathbf{v}}_{i}\right],
\end{aligned}
$$

so that

$$
\begin{aligned}
\underline{\underline{F}}\left(k, \mathbf{r}, \mathbf{r}^{\prime}\right)= & \frac{i k}{4 \pi}\left(\hat{\mathbf{n}} \cdot \hat{\mathbf{k}}_{i}\right)\left[R_{h} \hat{\mathbf{h}}_{i} \hat{\mathbf{h}}_{i}-R_{v} \hat{\mathbf{v}}_{i} \hat{\mathbf{v}}_{i}\right] \\
& +\frac{i k}{4 \pi}\left[\underline{\underline{I}}-\hat{\mathbf{k}}_{i} \hat{\mathbf{k}}_{i}\right] \cdot\left[\left(\hat{\mathbf{k}}_{r} \hat{\mathbf{n}}\right) \cdot \underline{\underline{R}}-\left(\hat{\mathbf{n}} \cdot \hat{\mathbf{k}}_{r}\right) \underline{\underline{R}}\right] .
\end{aligned}
$$

Note from (A.6) that

$$
\left(\widehat{\mathbf{n}} \cdot \hat{\mathbf{k}}_{i}\right)=-\left(\widehat{\mathbf{n}} \cdot \hat{\mathbf{k}}_{r}\right) .
$$

Note also from (A.5), (A.8), and (A.10) that

$$
\hat{\mathbf{n}} \cdot \underline{\underline{R}}=R_{v}\left(\hat{\mathbf{n}} \cdot \hat{\mathbf{v}}_{r}\right) \hat{\mathbf{v}}_{i}=R_{v}\left(\hat{\mathbf{n}} \cdot \widehat{\mathbf{v}}_{i}\right) \hat{\mathbf{v}}_{i}
$$

Thus expanding the last term in (A.25),

$$
\begin{aligned}
& {\left[\left(\hat{\mathbf{k}}_{r} \hat{\mathbf{n}}\right) \cdot \underline{\underline{R}}-\left(\hat{\mathbf{n}} \cdot \hat{\mathbf{k}}_{r}\right) \underline{\underline{R}}\right]} \\
& =\left[R_{v}\left(\hat{\mathbf{n}} \cdot \widehat{\mathbf{v}}_{r}\right) \hat{\mathbf{k}}_{r} \hat{\mathbf{v}}_{i}-\left(\hat{\mathbf{n}} \cdot \hat{\mathbf{k}}_{r}\right) \underline{\underline{R}}\right] \\
& =R_{v}\left[\left(\hat{\mathbf{n}} \cdot \hat{\mathbf{v}}_{r}\right) \hat{\mathbf{k}}_{r}-\left(\hat{\mathbf{n}} \cdot \hat{\mathbf{k}}_{r}\right) \widehat{\mathbf{v}}_{r}\right] \widehat{\mathbf{v}}_{i}+R_{h}\left(\hat{\mathbf{n}} \cdot \hat{\mathbf{k}}_{i}\right) \hat{\mathbf{h}}_{i} \hat{\mathbf{h}}_{i} \\
& =R_{v}\left[\hat{\mathbf{n}} \times \hat{\mathbf{k}}_{r} \times \widehat{\mathbf{v}}_{r}\right] \hat{\mathbf{v}}_{i}+R_{h}\left(\hat{\mathbf{n}} \cdot \hat{\mathbf{k}}_{i}\right) \hat{\mathbf{h}}_{i} \hat{\mathbf{h}}_{i} \\
& =-R_{v}\left[\hat{\mathbf{n}} \times \hat{\mathbf{h}}_{i}\right] \widehat{\mathbf{v}}_{i}+R_{h}\left(\hat{\mathbf{n}} \cdot \hat{\mathbf{k}}_{i}\right) \hat{\mathbf{h}}_{i} \hat{\mathbf{h}}_{i} \\
& =-R_{v}\left[-\hat{\mathbf{n}} \times \hat{\mathbf{k}}_{i} \times \widehat{\mathbf{v}}_{i}\right] \widehat{\mathbf{v}}_{i}+R_{h}\left(\hat{\mathbf{n}} \cdot \hat{\mathbf{k}}_{i}\right) \hat{\mathbf{h}}_{i} \hat{\mathbf{h}}_{i} \\
& =-R_{v}\left(\hat{\mathbf{n}} \cdot \hat{\mathbf{k}}_{i}\right) \widehat{\mathbf{v}}_{i} \hat{\mathbf{v}}_{i}+R_{h}\left(\hat{\mathbf{n}} \cdot \hat{\mathbf{k}}_{i}\right) \hat{\mathbf{h}}_{i} \hat{\mathbf{h}}_{i}+R_{v}\left(\hat{\mathbf{n}} \cdot \widehat{\mathbf{v}}_{i}\right) \hat{\mathbf{k}}_{i} \widehat{\mathbf{v}}_{i} .
\end{aligned}
$$

Therefore, using (A.28),

$$
\begin{aligned}
& {\left[\underline{\underline{I}}-\hat{\mathbf{k}}_{i} \hat{\mathbf{k}}_{i}\right] \cdot\left[\left(\hat{\mathbf{k}}_{r} \hat{\mathbf{n}}\right) \cdot \underline{\underline{R}}-\left(\hat{\mathbf{n}} \cdot \hat{\mathbf{k}}_{r}\right) \underline{\underline{R}}\right]} \\
& =-R_{v}\left(\widehat{\mathbf{n}} \cdot \hat{\mathbf{k}}_{i}\right) \hat{\mathbf{v}}_{i} \hat{\mathbf{v}}_{i}+R_{h}\left(\hat{\mathbf{n}} \cdot \hat{\mathbf{k}}_{i}\right) \hat{\mathbf{h}}_{i} \hat{\mathbf{h}}_{i} .
\end{aligned}
$$


Combining (A.28) with (A.29) finally yields

$$
\underline{\underline{F}}=\frac{i k}{2 \pi}\left(\widehat{\mathbf{n}} \cdot \widehat{\mathbf{k}}_{i}\right)\left[R_{h} \hat{\mathbf{h}}_{i} \hat{\mathbf{h}}_{i}-R_{v} \widehat{\mathbf{v}}_{i} \widehat{\mathbf{v}}_{i}\right] .
$$

It is perhaps worth mentioning that the expression (A.30) has a level of simplicity rarely encountered in the literature, and makes quite transparent the dependence of reflectivity on surface properties in the tangent plane approximation.

\section{B. SPOTLIGHT SAR AND THE POLAR FORMAT ALGORITHM}

We consider formation of a spotlight SAR image using a transmitted linear FM chirp pulse. The $n^{\text {th }}$ pulse, with chirp rate $\beta$, has the form

$p(\hat{t})=\exp \left(-i \omega_{o} n T\right)\left\{\operatorname{Rect}\left[\frac{\hat{t}}{T_{p}}\right] \exp \left(-i \omega_{o} \hat{t}\right) \exp \left(-i \beta \hat{t}^{2}\right)\right\}$.

Choosing a negative frequency, rather than a traditional positive frequency, permits an easier connection with the scattering theory analysis: the choice makes no significant difference to the ultimate expressions for coherence. Following $[8,9]$, the fast time $T$ is the interpulse period and $T_{p}$ is the pulse duration. Using (5a) and (B.1), the received signal from a differential surface element, with delay $\tau$, may be written as

$$
\begin{aligned}
& \Delta \underline{\underline{s}}(u(n), \hat{t}) \\
& \quad=\underline{\underline{a}} \operatorname{Rect}\left[\frac{\hat{t}-\tau}{T_{p}}\right] \exp \left(-i \omega_{o}(t-\tau)\right) \exp \left(-i \beta(\hat{t}-\tau)^{2}\right),
\end{aligned}
$$

where $\underline{\underline{a}}=\underline{\underline{\Phi}}\left(\omega_{o}, x, y, u\right) d x d y$ is the reflectivity determined in Appendix A. The first stage in the $\mathrm{P} f a$ is the "deramp" or "dechirp" procedure $[8,9]$, which involves mixing of the received signal with a reference signal to reduce the required $\mathrm{A} / \mathrm{D}$ sample rate. The reference signal, the same for each polarimetric channel, is

$$
\begin{aligned}
& s_{\mathrm{ref}}(u(n), \hat{t}) \\
& \quad=\operatorname{Rect}\left[\frac{\hat{t}-\tau_{o}}{T_{p}}\right] \exp \left(i \omega_{o}\left(t-\tau_{o}\right)\right) \exp \left(i \beta\left(\hat{t}-\tau_{o}\right)^{2}\right),
\end{aligned}
$$

where the delay time, $\tau_{o}(u)=2 r(u) / c$, is the round-trip time to scene centre. After mixing with the reference signal, an intermediate signal results, given by

$$
\begin{aligned}
\Delta \underline{\underline{s}}_{i}(u(n), \hat{t}) & \\
\approx & \underline{\underline{a}} \operatorname{Rect}\left[\frac{\hat{t}-\tau}{T_{p}}\right] \exp \left(i\left(\omega_{o}+2 \beta\left(\hat{t}-\tau_{o}\right)\right)\left(\tau-\tau_{o}\right)\right) \\
& \times \exp \left(-i \beta\left(\tau-\tau_{o}\right)^{2}\right) .
\end{aligned}
$$

Note that in writing (B.4) we have made the tacit approximation that the width of the "Rect" function product is the same as the width of the original "Rect" function. This approximation will be valid close to the scene centre. The $\mathrm{P} f a$ treatment is more conveniently discussed in terms of range variables, rather than fast times, and defining the range quantities

$$
r^{\prime}=\frac{c \hat{t}}{2}, \quad R=\frac{c \tau}{2}, \quad r=\frac{c \tau_{o}}{2}, \quad R_{p}=\frac{c T_{p}}{2}
$$

permits the differential intermediate signal of (B.4) to be written as

$$
\begin{aligned}
\Delta \underline{\underline{s}}(i), r)= & \underline{\underline{a}} \operatorname{Rect}\left[\frac{r^{\prime}-R(u)}{R_{p}}\right] \\
& \times \exp \left(i 2\left\{k_{o}+\frac{4 \beta}{c^{2}}\left[r^{\prime}-r(u)\right]\right\}[R(u)-r(u)]\right) \\
& \times \exp \left(-i \frac{4 \beta}{c^{2}}[R(u)-r(u)]^{2}\right) .
\end{aligned}
$$

The derivation of the $\mathrm{P} f a$ proceeds by showing how the intermediate signal can be considered as a sample of the Fourier transform of a "reflectivity" function. Given that the differential element of surface is at position $\mathbf{s}=\hat{\boldsymbol{s}} \hat{\boldsymbol{s}}$, start by expanding the range difference $R(u)-r(u)$ about the instantaneous range to scene centre:

$$
\begin{aligned}
R(u) & =|\mathbf{r}(u)-\mathbf{s}| \\
& =r(u)\left[\left(\widehat{\mathbf{n}}(u)-\frac{s}{r(u)} \hat{\mathbf{s}}\right) \cdot\left(\hat{\mathbf{n}}(u)-\frac{s}{r(u)} \hat{\mathbf{s}}\right)\right]^{1 / 2} \\
& \approx r(u)-s(\hat{\mathbf{n}}(u) \cdot \hat{\mathbf{s}})+\frac{s^{2}\left(1-(\hat{\mathbf{n}}(u) \cdot \widehat{\mathbf{s}})^{2}\right)}{2 r(u)},
\end{aligned}
$$

where the unit vector $\widehat{\mathbf{n}}(u)=\mathbf{r}(u) / r(u)$. Define a wavenumber $\kappa(u, r)$ such that

$$
\kappa(u, r)=2 k_{o}+\frac{8 \beta}{c^{2}}\left(r^{\prime}-r(u)\right) .
$$

The phase of the intermediate signal may be expanded using (B.7) and (B.8), and keeping leading terms, one finds that

$$
\begin{aligned}
& \phi_{i}(u, r) \\
& \quad \approx-s \kappa(u, r)(\hat{\mathbf{n}} \cdot \widehat{\mathbf{s}})+\frac{\kappa(u, r) s^{2}}{2 r(u)}\left(1-(\hat{\mathbf{n}} \cdot \widehat{\mathbf{s}})^{2}\right)-\frac{4 \beta s^{2}}{c^{2}}(\widehat{\mathbf{n}} \cdot \widehat{\mathbf{s}})^{2} .
\end{aligned}
$$

This expression can be seen to be in agreement with $[8$, (B.19)] if we set $(\hat{\mathbf{n}} \cdot \widehat{\mathbf{s}})=\sin (\theta+\gamma)$, with $\theta$ and $\gamma$ defined in [8], and account for the change to negative frequency by multiplying (B.9) by -1 .

The second term in (B.9) is referred to in the texts as the range curvature term, whilst the last term is known as the residual deramp phase. We note that both the range curvature and residual deramp phases may be ignored if the spatial extent of the image area is limited at sufficiently high range 
[8]. In these circumstances, the phase of the intermediate signal is simply approximated by

$$
\begin{aligned}
\phi_{i}(u, r) & \approx-\boldsymbol{\kappa}(u, r) \cdot \mathbf{s}, \\
\boldsymbol{\kappa}(u, r) & =\kappa(u, r) \hat{\mathbf{n}}(u) .
\end{aligned}
$$

The intermediate signal for the differential area may thus be expressed as

$$
\Delta \underline{\underline{s}}_{i p}\left(u_{p}, r\right)=\underline{\underline{a}}_{p} \operatorname{Rect}\left[\frac{r-R\left(u_{p}\right)}{R_{p}}\right] \exp \left(i \phi_{i p}\left(u_{p}, r\right)\right) .
$$

To proceed further, we consider imaging using a circular platform trajectory with constant slant-range radius $r$ to scene centre, and radial projection in the imaging plane $r \sin \theta$. The plane of the orbit is chosen parallel to the imaging plane and as a consequence the angle of incidence with the imaging plane, $\theta$, is a constant of the platform trajectory. The alongtrack variable, $u$, is now specified as the platform displacement in the azimuthal direction $\hat{\mathbf{x}}$. Let

$$
\kappa_{\rho}=\left(\kappa_{x}^{2}+\kappa_{y}^{2}\right)^{1 / 2}=\kappa \sin \theta
$$

then from (B.8),

$$
r^{\prime}=\frac{c^{2}}{8 \beta}\left[\frac{\left(\kappa_{x}^{2}+\kappa_{y}^{2}\right)^{1 / 2}}{\sin \theta}-2 k_{o}\right]+r
$$

and from (B.10),

$$
u=\frac{r \kappa_{x} \sin \theta}{\left(\kappa_{x}^{2}+\kappa_{y}^{2}\right)^{1 / 2}}
$$

The platform ground-range coordinate may then be identified as

$$
y=\frac{r \kappa_{y} \sin \theta}{\left(\kappa_{x}^{2}+\kappa_{y}^{2}\right)^{1 / 2}}
$$

Given that

$$
\kappa=\left(\kappa_{x}^{2}+\kappa_{y}^{2}+\kappa_{z}^{2}\right)^{1 / 2}
$$

from (B.12), it is clear that

$$
\kappa_{z}=\frac{\left(\kappa_{x}^{2}+\kappa_{y}^{2}\right)^{1 / 2}}{\tan \theta}
$$

The phase of our differential, intermediate signal is thus revealed as

$$
\begin{aligned}
\phi_{i}\left(\kappa_{x}, \kappa_{y}\right) & \approx-\kappa\left(\frac{u}{r} s_{x}+\frac{y}{r} s_{y}+\frac{h}{r} s_{z}\right) \\
& =-\left(\kappa_{x} s_{x}+\kappa_{y} s_{y}+\kappa_{z} s_{z}\right) \\
& \equiv-\left(\kappa_{x} x+\kappa_{y} y+\kappa_{z} h(x, y)\right) .
\end{aligned}
$$

Referring to (B.11) for the intermediate signal for the differential area reveals that the intermediate signal represents only a sample of the Fourier transform of the reflectivity, part of an annulus in the $\left(\kappa_{x}, \kappa_{y}\right)$ domain. To see this, consider the returns from the centre of the scene at slant range $r$; then from (B.13) and (B.11),

$$
\left|\kappa-2 k_{o}\right| \leq \frac{4 \beta}{c^{2}} R_{p}=\frac{2 \beta}{c} T_{p}=2 \pi \frac{2 B}{c},
$$

where $B$ is the radar bandwidth. For a point displaced from the scene centre by an amount $\delta R=c^{2} \delta \kappa / 8 \beta$ in slant range, the available sample width is reduced by an amount $\delta \kappa$. So it is possible to form the Fourier transform only by integration over a region sampled by the spotlight SAR system: usually a rectangular region of the $\left(\kappa_{x}, \kappa_{y}\right)$ domain centred at or close to $\kappa=2 k_{o}$. When forming a second image to create a pair for coherence calculations, the region of integration may be chosen to be common to both samples. In either case, the FT of the intermediate, differential signal may be formed as

$$
\begin{aligned}
& \Delta \underline{\underline{\Omega}}=\int_{-\Delta \kappa_{x}}^{\Delta \kappa_{x}} \int_{2 k_{o} \sin \theta-\Delta \kappa_{y}}^{2 k_{o} \sin \theta+\Delta \kappa_{y}}\left(u\left(\kappa_{x}, \kappa_{y}\right), r\left(\kappa_{x}, \kappa_{y}\right)\right) \exp \left(i \kappa_{x} x^{\prime}\right) \\
& \times \exp \left(i \kappa_{y} y^{\prime}\right) d \kappa_{x} d \kappa_{y}
\end{aligned}
$$

which, using (B.11) and (B.20), becomes

$$
\begin{aligned}
\Delta \underline{\underline{\Omega}=\int_{-\Delta \kappa_{x}}^{\Delta \kappa_{x}} \int_{2 k_{o} \sin \theta-\Delta \kappa_{y}}^{2 k_{o} \sin \theta+\Delta \kappa_{y}} \underline{\underline{a}} \exp \left(-i \kappa_{z} h(x, y)\right)} \\
\quad \times \exp \left(i \kappa_{x}\left(x^{\prime}-x\right)\right) \exp \left(i \kappa_{y}\left(y^{\prime}-y\right)\right) d \kappa_{x} d \kappa_{y} .
\end{aligned}
$$

In the narrow bandwidth approximation, $\underline{a}=\underline{\Phi}\left(\omega_{o}, x, y\right.$, $\left.u_{o}\right) d x d y$, and for the rough surface, $s_{z}=\overline{\bar{h}}(x, y)$, thus applying the FT to the full intermediate signal yields

$$
\begin{aligned}
& \underline{\underline{\Omega}}\left(x^{\prime}, y^{\prime}\right) \\
& =\iint_{S} \int_{-\Delta \kappa_{x}}^{\Delta \kappa_{x}} \int_{2 k_{o} \sin \theta-\Delta \kappa_{y}}^{2 k_{o} \sin \theta+\Delta \kappa_{y}}\left\{\underline{\underline{\Phi}}\left(\omega_{o}, x, y, u_{o}\right) e^{-i \kappa_{z} h(x, y)} e^{i \kappa_{x}\left(x^{\prime}-x\right)}\right. \\
& \left.\quad \times e^{i \kappa_{y}\left(y^{\prime}-y\right)} d \kappa_{x} d \kappa_{y}\right\} d x d y .
\end{aligned}
$$

The insistence of narrow aperture as well as narrow bandwidth may be overcome simply by replacing the approximate reflectivity $\Phi\left(\omega_{o}, x, y, u_{o}\right)$ in (B.22) with the full reflectivity in the narrow $\bar{b}$ andwidth approximation $\Phi\left(\omega_{o}, x, y, u\left(\kappa_{x}, \kappa_{y}\right)\right)$. In the absence of range curvature and deramp phase errors (B.22) is an accurate representation of a spotlight SAR pixel value focused at $\left(x^{\prime}, y^{\prime}\right)$ in the imaging plane in the narrow bandwidth/aperture approximation. The validity of the narrow aperture and bandwidth approximations is discussed in the main text. 


\section{ACKNOWLEDGMENT}

This work has been supported by the Defence Science and Technology Organisation of Australia.

\section{REFERENCES}

[1] M. Preiss, D. Gray, and N. Stacy, "Change detection in repeat pass interferometric synthetic aperture radar," in Proc. International Conference on Radar, pp. 103-109, Adelaide, SA, Australia, September 2003.

[2] M. Preiss, D. Gray, and N. Stacy, "A change detection technique for repeat pass interferometric SAR," in Proc. IEEE International Geoscience and Remote Sensing Symposium (IGARSS '03), vol. 2, pp. 938-940, Toulouse, France, July 2003.

[3] D. G. Corr and A. Rodrigues, "Coherent change detection of vehicle movements," in Proc. IEEE International Geoscience and Remote Sensing Symposium (IGARSS '98), vol. 5, pp. 2451-2453, Seattle, Wash, USA, July 1998.

[4] N. Stacy, M. Preiss, and M. L. Williams, "Repeat-pass coherence measurements with fully polarimetric X-band SAR," in Proc. 5th European Conference on Synthetic Aperture Radar (EUSAR '04), Ulm, Germany, May 2004.

[5] A. K. Fung, Microwave Scattering and Emission Models and Their Applications, Artech House Remote Sensing Library, Artech House, Norwood, Mass, USA, 1994.

[6] F. T. Ulaby and C. Elachi, Radar Polarimetry for Geoscience Applications, Artech House Remote Sensing Library, Artech House, Norwood, Mass, USA, 1990.

[7] F. T. Ulaby, R. K. Moore, and A. K. Fung, Microwave Remote Sensing Active and Passive, Volume II, Radar Remote Sensing and Surface Scattering and Emission Theory, Remote Sensing Series, Artech House, Norwood, Mass, USA, 1986.

[8] C. V. Jakowatz Jr., D. E. Wahl, P. H. Eichel, D. C. Ghiglia, and P. A. Thompson, Spotlight-Mode Synthetic Aperture Radar: A Signal Processing Approach, Kluwer Academic, Boston, Mass, USA, 1996.

[9] W. G. Carrara, R. S. Goodman, and R. M. Majewski, Spotlight Synthetic Aperture Radar Signal Processing Algorithms, Artech House Remote Sensing Library, Artech House, Norwood, Mass, USA, 1995.

[10] H. A. Zebker and J. Villasenor, "Decorrelation in interferometric radar echoes," IEEE Trans. Geosci. Remote Sensing, vol. 30, no. 5, pp. 950-959, 1992.

[11] R. White and M. L. Williams, "Processing ISAR and spotlight SAR data to very high resolution," in Proc. IEEE International Geoscience and Remote Sensing Symposium (IGARSS '99), vol. 1, pp. 32-34, Hamburg, Germany, June-July 1999.

[12] H. Cantalloube and P. Dubois-Fernandez, "Airborne X-band SAR imaging with $10 \mathrm{~cm}$ resolution-technical challenge and preliminary results," in Proc. 5th European Conference on Synthetic Aperture Radar (EUSAR '04), vol. 1, pp. 271-274, Ulm, Germany, May 2004.

[13] J. A. Ogilvy, Theory of Wave Scattering from Random Rough Surfaces, IOP Publishing, Bristol, UK, 1991.

[14] E. I. Thorsos and D. R. Jackson, "Studies of scattering theory using numerical methods," Waves in Random Media, vol. 1, no. 3, pp. 165-190, 1991.

[15] F. J. Harris, "On the use of windows for harmonic analysis with the discrete fourier transform," Proc. IEEE, vol. 66, no. 1, pp. 51-83, 1978.

[16] C. Thiel, S. Gruenler, M. Herold, V. Hochschild, G. Jaeger, and M. Hellmann, "Interpretation and analysis of polarimetric $L$ band E-SAR-data for the derivation of hydrologic land surface parameters," in Proc. IEEE International Geoscience and
Remote Sensing Symposium (IGARSS '01), vol. 7, pp. 32153217, Sydney, NSW, Australia, July 2001.

[17] F. Gatelli, A. M. Guamieri, F. Parizzi, P. Pasquali, C. Prati, and F. Rocca, "The wavenumber shift in SAR interferometry," IEEE Trans. Geosci. Remote Sensing, vol. 32, no. 4, pp. 855-865, 1994.

[18] R. Bamler, "Interferometric stereo radargrammetry: absolute height determination from ERS-ENVISAT interferograms," in Proc. IEEE International Geoscience and Remote Sensing Symposium (IGARSS '00), vol. 2, pp. 742-745, Honolulu, Hawaii, USA, July 2000.

[19] R. Touzi, A. Lopes, J. Bruniquel, and P. W. Vachon, "Coherence estimation for SAR imagery," IEEE Trans. Geosci. Remote Sensing, vol. 37, no. 1, pp. 135-149, 1999.

[20] C. J. Oliver and S. Quegan, Understanding Synthetic Aperture Radar Images, Artech House Remote Sensing Library, Artech House, Norwood, Mass, USA, 1998.

[21] M. R. Foster and N. J. Guinzy, "The coefficient of coherence: its estimation and use in geophysical data processing," Geophysics, vol. 32, no. 4, pp. 602-616, 1967.

[22] C. H. Gierull, "Unbiased coherence estimator for SAR interferometry with application to moving target detection," Electronics Letters, vol. 37, no. 14, pp. 913-915, 2001.

[23] C. H. Gierull and I. C. Sikaneta, "Estimating the effective number of looks in interferometric SAR data," IEEE Trans. Geosci. Remote Sensing, vol. 40, no. 8, pp. 1733-1742, 2002.

[24] M. Preiss, D. Gray, and N. Stacy, "A change detection statistic for repeat pass interferometric SAR," in Proc. IEEE International Conference on Acoustics, Speech, and Signal Processing (ICASSP '03), vol. 5, pp. 241-244, Hong Kong, China, April 2003.

[25] M. Preiss, Detecting scene changes using synthetic aperture radar interferometry, Ph.D. thesis, School of Electrical and Electronic Engineering, University of Adelaide, Adelaide, SA, Australia, 2004.

[26] M. Preiss, D. Gray, and N. Stacy, "A log likelihood statistic for change detection in repeat pass interferometric SAR," in Proc. 5th European Conference on Synthetic Aperture Radar (EUSAR '04), Ulm, Germany, May 2004.

[27] M. L. Williams, M. Preiss, and N. Stacy, "Performance and modelling of coherent change detection with the ingara Xband PolSAR," in Proc. 5th European Conference on Synthetic Aperture Radar (EUSAR '04), Ulm, Germany, May 2004.

Mark L. Williams was born in Sidcup, Kent, England, in 1961. He obtained a B.S. degree in physics with computing in 1983, and Ph.D. degree in physics in 1987, both from the University of Kent, Canterbury. He has occupied teaching and research positions at the University of Kent, Canterbury, Universiti Brunei Darussalam, the National University of Singapore, and most recently the University of Sheffield, where he first be-

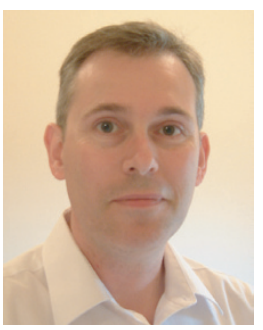
gan work in radar remote sensing at SCEOS in 1994. He moved to the Defence Research Agency (formerly the RSRE), Malvern, in 1996, surviving the metamorphosis of that establishment until 2002, working predominantly on synthetic aperture radar and the theoretical modelling of microwave backscatter. He moved to Australia in 2002 and now works for the Defence Science and Technology Organisation, Adelaide. His current research interests include the validation and exploitation of combined coherent scattering and SAR imaging models. 
Mark Preiss received the B.E. (honours) and Ph.D. degrees in electrical engineering from the University of Adelaide, Adelaide, Australia, in 1994 and 2004, respectively. From 1994 to 1996, he was with the Communications Division of the Australian Defence Science and Technology Organisation (DSTO), developing an HF channel simulator for HF modem testing. Since 1996, he has been with the Imaging Radar Systems

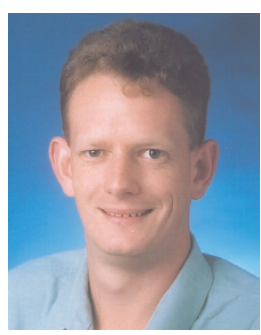
Group at the DSTO, supporting the ongoing development of the Ingara X-band synthetic aperture radar (SAR) and conducting research into imaging radar techniques. His research interests include SAR image formation, interferometric change detection techniques, and multichannel/multibaseline interferometric SAR. 